\title{
Aortic carboxypeptidase-like protein, a WNT ligand, exacerbates nonalcoholic steatohepatitis
}

\author{
Toshiaki Teratani, ${ }^{1}$ Kengo Tomita, ${ }^{2}$ Takahiro Suzuki, ${ }^{1}$ Hirotaka Furuhashi, ${ }^{2}$ Rie Irie, ${ }^{3}$ Makoto Nishikawa, ${ }^{4}$ Junji Yamamoto, ${ }^{4}$ \\ Toshifumi Hibi, ${ }^{1}$ Soichiro Miura, ${ }^{2}$ Tohru Minamino, ${ }^{5}$ Yuichi Oike, ${ }^{6}$ Ryota Hokari, ${ }^{2}$ and Takanori Kanai' \\ 'Division of Gastroenterology and Hepatology, Department of Internal Medicine, Keio University School of Medicine, Shinjuku-ku, Tokyo, Japan. 2Division of Gastroenterology and Hepatology, Department \\ of Internal Medicine, National Defense Medical College, Tokorozawa-shi, Saitama, Japan. ${ }^{3}$ Department of Pathology, National Center for Child Health and Development, Okura, Setagaya-ku, Tokyo, Japan \\ ${ }^{4}$ Department of Surgery, National Defense Medical College, Namiki, Tokorozawa-shi, Saitama, Japan. ${ }^{5}$ Department of Cardiovascular Biology and Medicine, Niigata University Graduate School of Medical \\ and Dental Sciences, Niigata, Japan. ${ }^{6}$ Department of Molecular Genetics, Graduate School of Medical Sciences, Kumamoto University, Honjo, Chuo-ku, Kumamoto, Japan.
}

Incidence of nonalcoholic steatohepatitis (NASH), which is considered a hepatic manifestation of metabolic syndrome, has been increasing worldwide with the rise in obesity; however, its pathological mechanism is poorly understood. Here, we demonstrate that the hepatic expression of aortic carboxypeptidase-like protein (ACLP), a glycosylated, secreted protein, increases in NASH in humans and mice. Furthermore, we elucidate that ACLP is a ligand, unrelated to WNT proteins, that activates the canonical WNT pathway and exacerbates NASH pathology. In the liver, ACLP is specifically expressed in hepatic stellate cells (HSCs). As fatty liver disease progresses, ACLP expression is enhanced via activation of STAT3 signaling by obesity-related factors in serum. ACLP specifically binds to frizzled-8 and low-density lipoprotein-related receptor 6 to form a ternary complex that activates canonical WNT signaling. Consequently, ACLP activates HSCs by inhibiting PPAR $\gamma$ signals. HSC-specific ACLP deficiency inhibits fibrosis progression in NASH by inhibiting canonical WNT signaling in HSCs. The present study elucidates the role of canonical WNT pathway activation by ACLP in NASH pathology, indicating that NASH can be treated by targeting ACLP-induced canonical WNT pathway activation in HSCs.

\section{Introduction}

Nonalcoholic fatty liver disease (NAFLD) is considered the hepatic manifestation of metabolic syndrome. Currently, NAFLD is the most prevalent liver disease in the world, with an estimated 1 billion patients worldwide and prevalence of $25 \%-45 \%$ in western and some other countries $(1,2)$. Recently, the prevalence of NAFLD in children has also increased to $3 \%-10 \%$ (3). The increase in NAFLD coincides with that in obesity and obesityrelated diseases, both of which are considered risk factors for the onset and progression of NAFLD $(2,4)$. The pathology of NAFLD ranges from simple steatosis to steatohepatitis. While nonalcoholic fatty liver (NAFL), mainly comprising simple steatosis, has a favorable prognosis, nonalcoholic steatohepatitis (NASH) progressively develops into NASH-related advanced fibrosis or cirrhosis and triggers complications such as hepatocellular carcinoma (1). Therefore, it is important to elucidate the pathological mechanism by which NAFL develops into NASH. Although 2-hit (5) and multiple parallel-hit (6) hypotheses have been proposed, the exact underlying mechanism remains poorly understood. A recent large-scale longitudinal clinical study determined that among hepatic histopathological findings in NAFLD patients, only the liver fibrosis stage was independently associated with long-term

Authorship note: TT and KT contributed equally to this work as co-first authors. Conflict of interest: The authors have declared that no conflict of interest exists. Submitted: January 18, 2017; Accepted: February 1, 2018.

Reference information: J Clin Invest. 2018;128(4):1581-1596.

https://doi.org/10.1172/JCI92863. overall mortality, liver transplantation, and liver-related events (7). Therefore, elucidating the pathological mechanism of fibrosis progression in NAFLD and establishing a therapy targeting this mechanism are needed.

Activated hepatic stellate cells (HSCs) are a key source of extracellular matrix (ECM) in the pathology of liver fibrosis (8), thus playing a main role in disease progression. Meanwhile, the (canonical) WNT/ $\beta$-catenin-signaling pathway plays an important role in cell growth and differentiation regulation from early development into adulthood (9). In the canonical WNT pathway, WNT binds to a frizzled (FZD) family receptor and low-density lipoprotein receptor-related protein 5/6 (LRP 5/6) to relay signals (9). The canonical WNT pathway inhibits adipogenesis (10) and is believed to be involved in the pathological mechanism that links obesity and obesity-associated metabolic complications (11). In addition, the canonical WNT pathway has been recently reported to promote HSC activation by inhibiting adipogenic gene expression (12). Similarly, in the pathology of NAFLD progression, it is assumed that factors in obesity and metabolic syndrome, acting as background factors, modify the pathological mechanism of HSC activation via the canonical WNT pathway.

Aortic carboxypeptidase-like protein (ACLP) is a glycosylated protein secreted into the ECM by smooth muscle cells and lung fibroblasts (13). Whether or not ACLP is produced in the liver tissues in hepatic diseases is unknown, as are the function of ACLP and its role in hepatic diseases. In addition, although ACLP harbors an N-terminal signal peptide, a discoidin-like domain, and an inactive carboxypeptidase domain and belongs to the metallocar- 
boxypeptidase gene family, it is catalytically inactive and its functions are not well understood (13). ACLP-null mice experience gastroschisis, which often results in perinatal death (14); therefore, it has been impossible to analyze the involvement of ACLP in pathology using ACLP-null mice on a pure genetic background. Thus, conditional ACLP-KO mice should be developed for analysis of ACLP function in pathology.

In the present study, we demonstrated that ACLP is a ligand that binds specifically to FZD8 and LRP6 receptors, forming a ternary complex activating the canonical WNT pathway. In the liver, ACLP localized to the HSCs, and its expression increased with the onset and progression of NAFLD in both humans and mice. Using newly developed HSC-specific ACLP-KO mice and human liver tissue samples, we analyzed the role of ACLP in the pathological mechanism of NAFLD progression. The present study elucidated details of the pathological mechanism of NAFLD progression, in which obesity-related factors, acting as background factors, promote the production of ACLP in HSCs, thereby activating the HSCs through the activation of the canonical WNT pathway. In addition, we present a detailed characterization of ACLP as a ligand of the canonical WNT pathway.

\section{Results}

ACLP is expressed specifically in HSCs, and its expression is enhanced as NAFLD progresses. We found that hepatic ACLP expression significantly increased as NAFLD progressed (Figure 1A). Immunofluorescence double-staining of human liver samples for ACLP and the HSC markers (glial fibrillary acidic protein [GFAP] and desmin) revealed that ACLP is expressed specifically in HSCs (Figure 1A and Supplemental Figures 1 and 2; supplemental material available online with this article; https://doi.org/10.1172/ JCI92863DS1). In addition, ACLP expression in the HSCs increased significantly as NAFLD progressed (Figure 1, A and B).

As these results indicated that ACLP might be involved in the pathology of NAFLD progression, we analyzed the pathological mechanism of NAFLD in detail in mutant mice. First, we generated ACLP-floxed $\left(A c l p^{f / f l}\right)$ mice (Supplemental Figure 3). Next, we generated CRE Tg mice in which CRE expression was driven by the GFAP promoter ( $G f a p-C r e^{T g}$ mice). Finally, Gfap-Cre ${ }^{T g}$ mice were bred with $A c l p^{f / f l}$ mice to generate HSC-specific ACLP-KO $\left(A c l p^{H S C-K O}\right)$ mice (Supplemental Figure 3$)$. CRE expression was specifically observed in the livers of $A c l p^{H S C-K O}$ mice, and this localization was consistent with the expression of GFAP (Supplemental Figure 3). Immunofluorescence double-staining of mouse liver samples for ACLP and GFAP or desmin revealed that ACLP is expressed specifically in HSCs (Figure 1C and Supplemental Figures 4 and 5). ACLP expression in the liver disappeared in the $A c l p^{H S C-K O}$ mice (Figure 1, B and C), as did ACLP expression in HSCs (Figure 1, C and D, and Supplemental Figures 3-5). Mice fed a high fat/high cholesterol (HFC) diet for 24 weeks develop NASH through NAFL (15). When $A c l p^{f / f l}$ and $A c l p^{H S C-K O}$ mice were fed an HFC diet for 24 weeks, hepatic ACLP expression increased over time in $A c l p^{f l / f l}$, but not $A c l p^{H S C-K O}$, mice (Figure 1B). Immunofluorescence double-staining of ACLP and GFAP or desmin also showed that, in the livers of $A c l p^{f / f l}$ mice, ACLP was specifically expressed in HSCs and its expression increased as NAFLD progressed; however, Aclp ${ }^{H S C-K O}$ mice showed no ACLP expression (Figure $1 \mathrm{C}$ and
Supplemental Figures 4 and 5). In $A c l p^{f l f l}$ mice, ACLP expression in HSCs increased as NAFLD progressed, whereas in $A c l p^{H S C-K O}$ mice, HSCs were ACLP deficient (Figure 1D). We confirmed that ACLP was not expressed in other liver cells, such as hepatocytes, Kupffer cells, bile duct cells, and ductular-appearing cells (Supplemental Figures 6-8).

HSC-specific ACLP deficiency suppresses progression of liver fibrosis in NASH. Both Aclp $p^{f / f l}$ and Aclp $p^{H S C-K O}$ mice on an HFC diet developed NAFL at 4 weeks, which progressed to NASH at 24 weeks (Figure 2A). Examination of the NASH pathology revealed that, compared with $A c l p^{f l f l}$ mice, $A c l p^{H S C-K O}$ mice had significantly suppressed liver fibrosis suppression (Figure 2A). Moreover, Aclp ${ }^{H S C-K O}$ mice demonstrated significantly lower expression of the fibrosis markers collagen $1 \alpha 1$ and $1 \alpha 2$ in NASH livers than Aclpt/fl mice (Figure 2B). Similarly, expression of the HSC activation marker $\alpha$ SMA was significantly suppressed in Aclp ${ }^{H S C-K O}$ as compared with $A c l p^{f l / f l}$ mice (Figure 2, A and B).

The murine model of NASH had significantly elevated serum alanine aminotransferase (ALT), cholesterol, and triglyceride (TG) compared with a control group fed a normal diet; however, no significant differences were observed between $A c l p^{H S C-K O}$ and $A c l p^{f / f l}$ mice (Figure 2C and Supplemental Figure 9). Similarly, the NASH model demonstrated significantly higher TG accumulation in the liver, fasting blood glucose, and insulin levels than the control, while $A c l p^{H S C-K O}$ and $A c l p^{f / f l}$ mice did not differ from each other in these aspects (Figure 2, A and C, and Supplemental Figure 9). To assess insulin sensitivity and resistance, we conducted glucose tolerance tests (GTTs), insulin tolerance tests (ITTs), and pyruvate tolerance tests (PTTs). While significant differences were observed between the normal diet control and NASH groups in all tests, no significant differences were observed between $A c l p^{H S C-K O}$ and $A c l p^{f / f l}$ mice (Supplemental Figure 9).

Immunohistochemical staining for F4/80, a Kupffer cell/ macrophage marker, was used to assess macrophage infiltration into the liver. NASH mice demonstrated significantly higher macrophage infiltration than the controls; however, no significant difference was observed between $A c l p^{H S C-K O}$ and $A c l p^{f l / f l}$ mice (Supplemental Figure 10). Hepatic mRNA expression of Adgre1, Tnf, and $C d 68$ was significantly higher in NASH than in control mice, while no significant differences were found between $A c l p^{H S C-K O}$ and $A c l p^{f l f l}$ mice (Supplemental Figure 10). Together, the results demonstrate that ACLP, which is expressed specifically in HSCs, and more intensely so as NAFLD progresses, is involved in the progression of liver fibrosis in NASH via HSC activation.

ACLP promotes liver fibrosis in NASH by activating the canonical WNT pathway in HSCs. HSC activation has recently been reported to correlate with canonical WNT pathway activation $(12,16)$. Therefore, we examined mRNA expression of Axin2, $M y c$, and Ccnd1, which are downstream targets of this pathway, in HSCs from NASH model mice. In NASH, all tested molecules showed markedly higher expression in HSCs of $A c l p^{f / f l}$ mice than in those of control mice, while their expression did not change in HSCs of Aclp ${ }^{H S C-K O}$ mice (Figure 3A). Western blotting as well as immunofluorescence double-staining of GFAP and $\beta$-catenin demonstrated a significant increase in $\beta$-catenin accumulation, specifically, in HSCs of $A c l p^{f / f l}$ mice with NASH, but not in those of $A c l p^{H S C-K O}$ mice (Figure 3, A and B, and Supplemental Figure 11). 
A
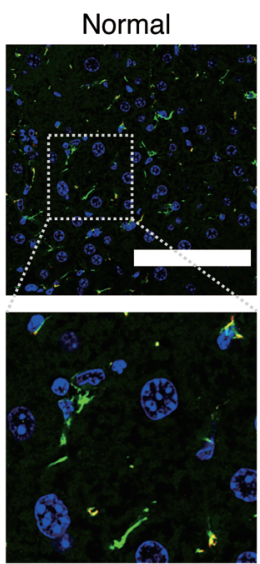

B
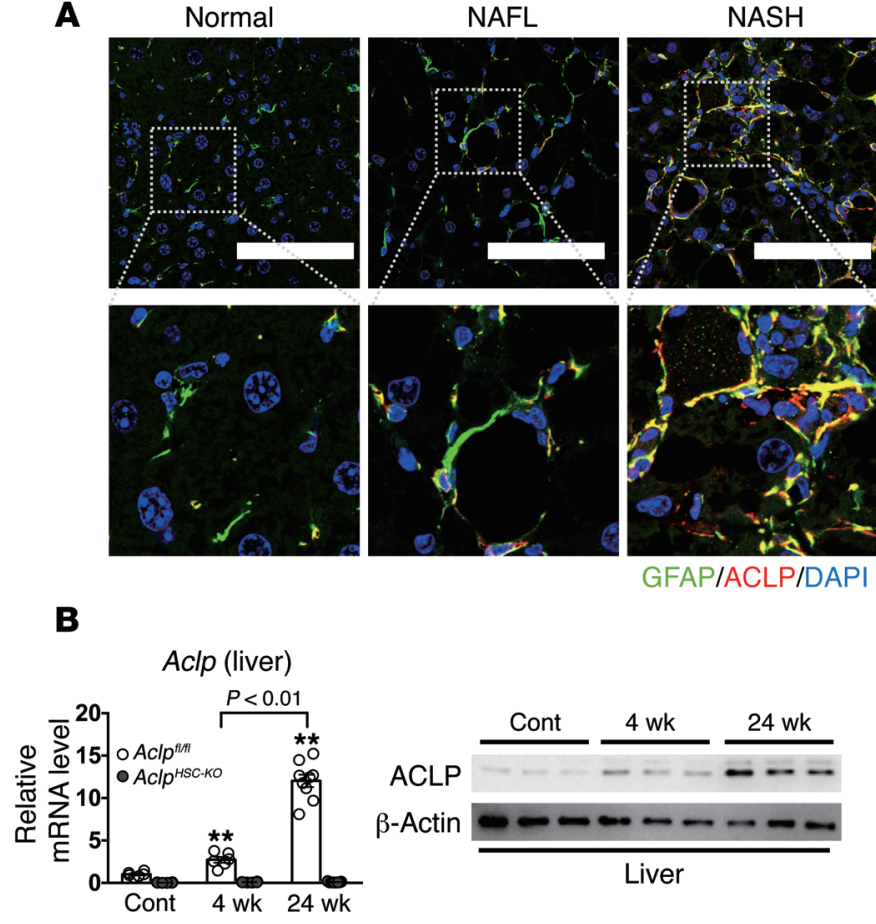

GFAP/ACLP/DAPI
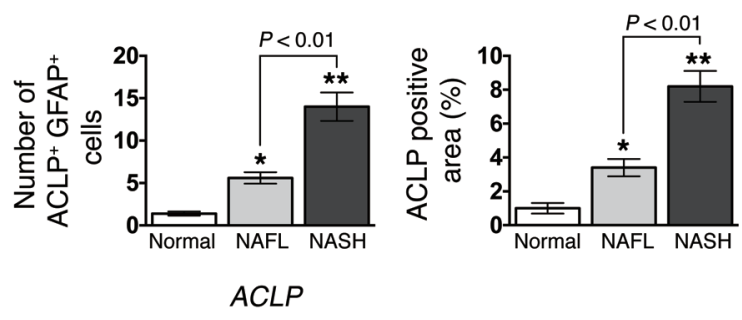

$A C L P$

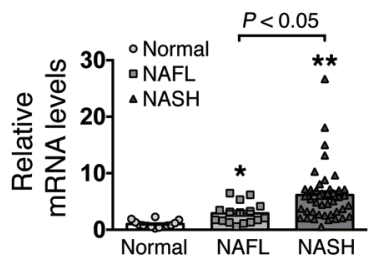

C
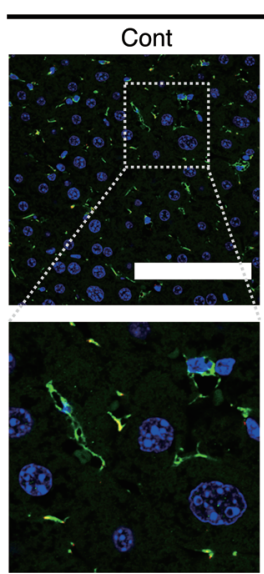

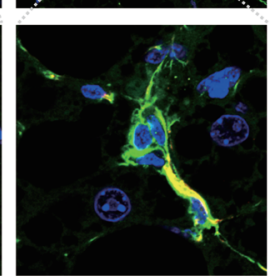

$A c \mid p p^{f|f|}$
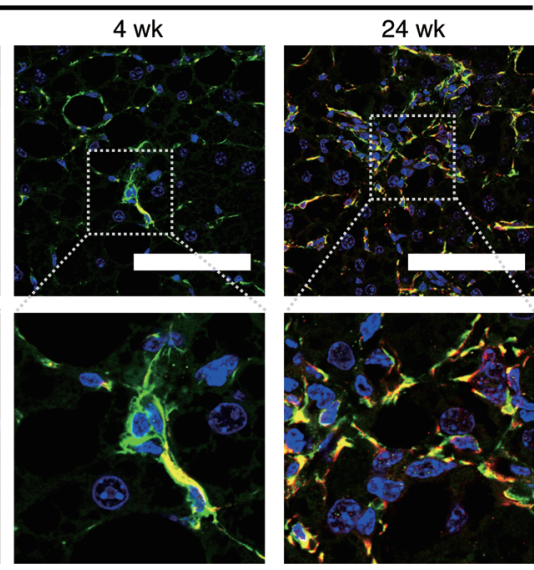

ACLP protein (liver)

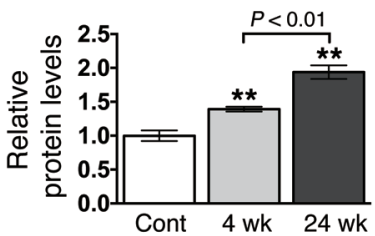

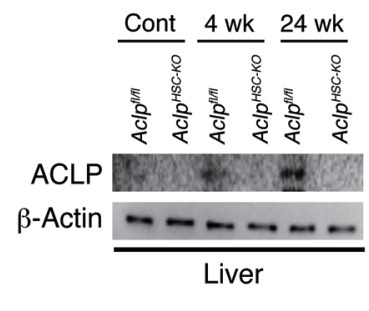

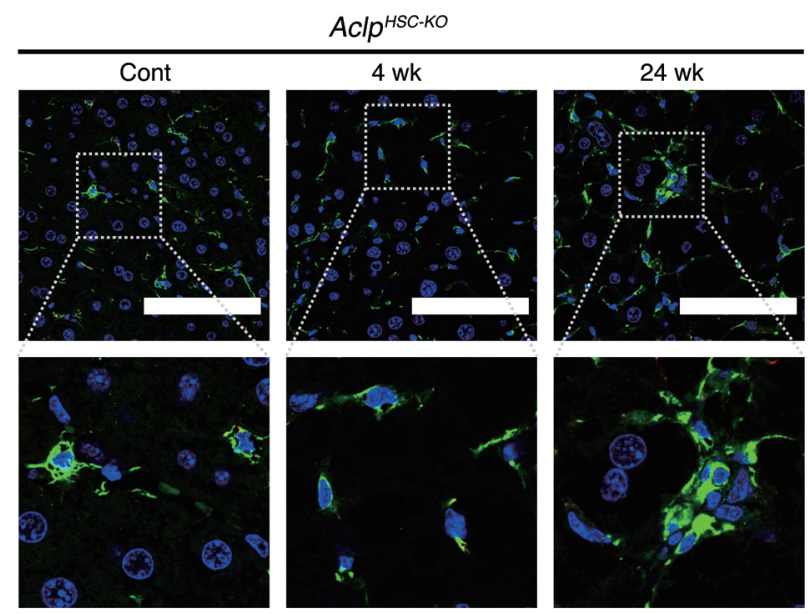

GFAP/ACLP/DAPI

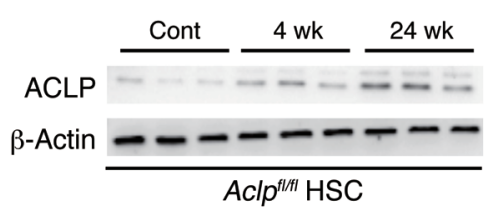

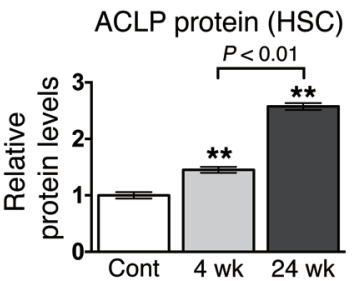

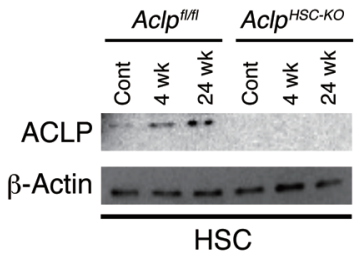

Figure 1. In the liver, ACLP is specifically expressed by HSCs, and its expression increases in both humans and mice as NAFLD progresses. (A) (Left panels) Representative immunofluorescence double-staining images of ACLP (red) and GFAP (green) in human liver tissue samples from controls ( $n=$ 14), NAFL patients $(n=16)$, and NASH patients $(n=44)$. Costained sites are shown in yellow. The nuclei were stained with DAPI (blue). Scale bars: 100 $\mu \mathrm{m}$. Single-channel images are shown in Supplemental Figure 1. (Right panels) Quantification of ACLP/GFAP double-positive cells, ACLP staining, and mRNA expression. ${ }^{* *} P<0.01$; ${ }^{*}<0.05$ vs. control (normal) liver samples. (B-D) Eight-week-old male Aclp ${ }^{f l f l}$ and $A c / p p^{H S C-K O}$ mice were fed an HFC diet for 4 weeks ( $n=6$ /group) or 24 weeks ( $n=9$ /group), or fed a control diet (CE-2) for 24 weeks ( $n=6 /$ group). (B) (Left panel) Hepatic Aclp mRNA expression levels. (Middle panel) Western blot for and quantification of hepatic ACLP expression in Aclp ${ }^{f / f l}$ mice. ${ }^{* *} P<0.01$ vs. Aclp ${ }^{f / f l}$ mice fed the control diet. (Right panel) Western blot for hepatic ACLP expression in Acl ${ }^{f l / f l}$ mice and $A c / p^{H S C-K O}$ mice. (C) Representative images of immunofluorescence double-staining of ACLP (red) and GFAP (green), with costained sites shown in yellow. The nuclei were stained with DAPI (blue). Scale bars: $100 \mu \mathrm{m}$. Single-channel images are shown in Supplemental Figure 4. (D) (Left panel) Western blot for and quantification of ACLP expression in HSCs in Aclp ${ }^{f l / f l}$ mice. ${ }^{* *} P<0.01$ vs. HSCs in $A c l p^{f l / f l}$ mice fed the control diet. (Right panel) Western blot for ACLP expression in HSCs in AcI $p^{f l / f l}$ mice and Aclp $p^{H S C-K O}$ mice. $P$ values obtained via 1-way ANOVA with Tukey's post hoc test. Data are shown as SEM. 
A
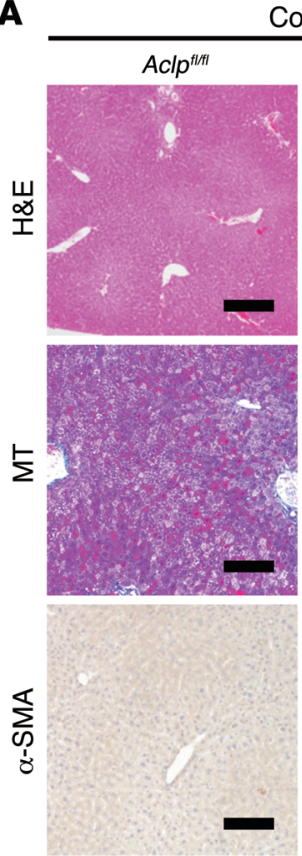

Masson trichrome

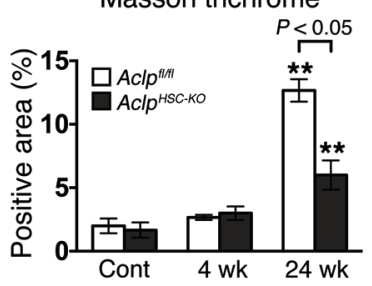

C

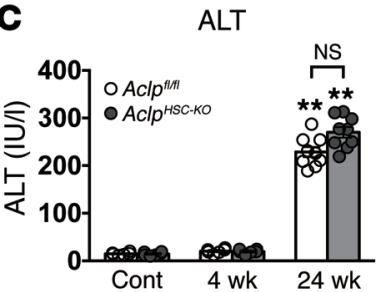

Cont
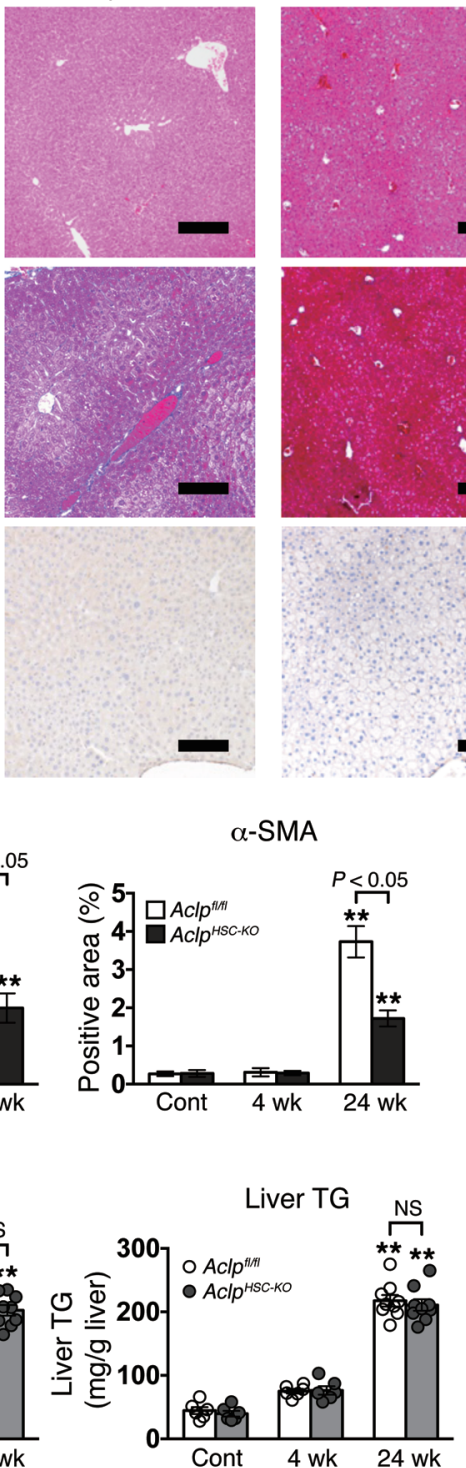

4 wk
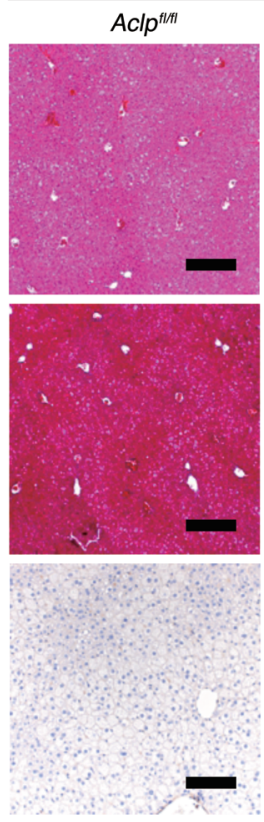

B
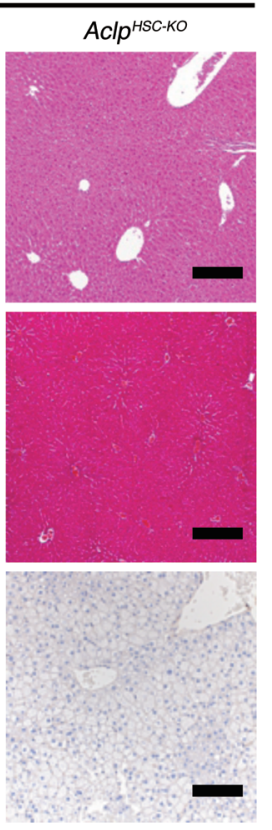

B

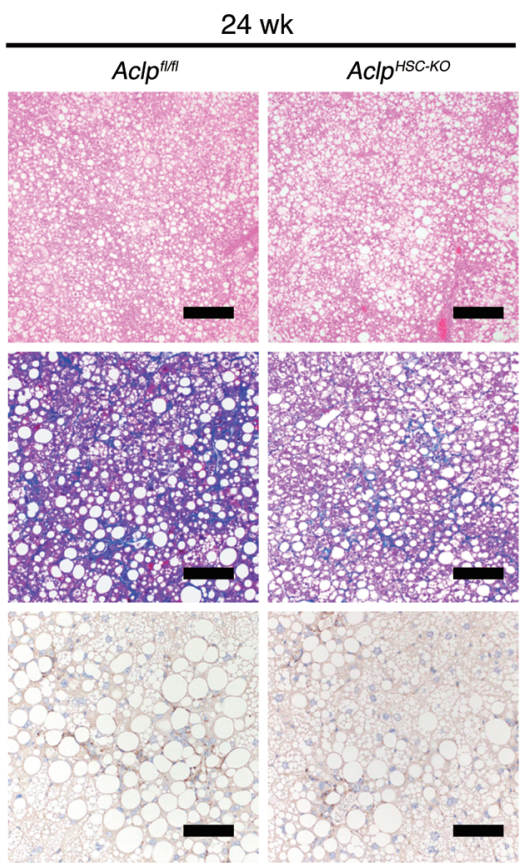

Col1a2 (liver)
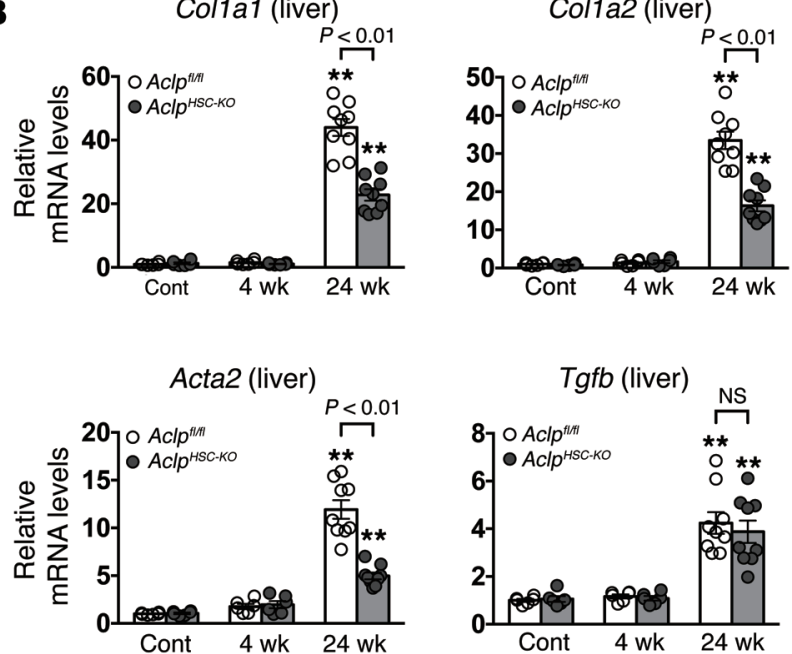

Figure 2. HSC-specific ACLP deficiency inhibits progression of liver fibrosis in murine NASH. (A-C) Eight-week-old male Aclp ${ }^{f l f l}$ and $A C l p^{H S C-K O}$ mice were fed an HFC diet for 4 weeks ( $n=6 /$ group) or 24 weeks ( $n=9 /$ group) or a control diet for 24 weeks ( $n=6 /$ group). (A) (Upper panels) Representative H\&Estained, MT-stained, and $\alpha$ SMA-immunostained liver samples. Scale bars: $100 \mu \mathrm{m}$ (H\&E-stained and MT-stained sections); $50 \mu \mathrm{m}$ ( $\alpha \mathrm{SMA-immunostained}$ sections). (Lower panels) Quantification of MT staining and aSMA immunostaining. (B) Hepatic expression of Col1a1, Col1a2, Acta2, and Tgfb mRNA. (C) Serum ALT activities and hepatic TG levels. ${ }^{*} P<0.01$ vs. Acl $p^{f l / f l}$ mice fed a control diet for 24 weeks. $P$ values obtained via 1 -way ANOVA with Tukey's post hoc test. Data are shown as SEM.

Aclp $p^{f / f l}$ mice with NASH showed significantly more ACLP/ $\beta$-catenin double-positive cells than the control group, and $\beta$-catenin was upregulated specifically in ACLP-positive cells in NASH (Figure $3 \mathrm{C}$ and Supplemental Figure 11).

Dickkopf protein 1 (DKK-1) is a negative regulator of canonical WNT signaling $(17,18)$. Therefore, we bred $A c l p^{H S C-K O}$ mice with collagen $1 \alpha 1$ promoter-DKK-1 Tg (Dkk-1 $\left.{ }^{T g}\right)$ mice (19) to prepare doublemutant $A c l p^{H S C-K O} D k k-1^{T g}$ mice. Aclp ${ }^{f l f l}, A c l p^{H S C-K O}, A c l p^{f / f l} D k k-1^{T g}$, and $A c l{ }^{H S C-K O} D k k-1{ }^{T g}$ mice were fed an HFC diet for 24 weeks to induce NASH. $A c l p^{f l f l} D k k-1^{T g}$ mice showed a significant decline in both NASH liver fibrosis and HSC activation in comparison with $A c l p^{f / f l}$ mice, with levels similar to those in $A c l p^{H S C-K O}$ mice (Sup- plemental Figure 12). No significant differences were observed in NASH fibrosis progression and HSC activation between Aclp $p^{f / f l}$ $D k k-1^{T g}$ and $A c l p^{H S C-K O} D k k-1^{T g}$ mice (Supplemental Figure 12). No differences were observed in the degree of liver steatosis or serum ALT among any of the NASH model groups (Supplemental Figure 12). Aclp $p^{f / f l}$ NASH model-derived HSCs demonstrated significantly $\left({ }^{* *} P<0.01 \mathrm{vs.} A c l p^{f / f l}\right.$ mice fed a control diet for 24 weeks in Supplemental Figure 12) higher mRNA expression of Axin2, Myc, and Ccnd1 than HSCs from the normal-diet control group. However, in $D k k-1^{T g}$-derived as well as $A c l p^{H S C-K O}$-derived HSCs, the expression of these WNT targets did not increase in NASH, and no significant differences were observed between $A c l p^{f / f l} D k k-1^{T g_{-}}$and $A c l p^{H S C-K O}$ 


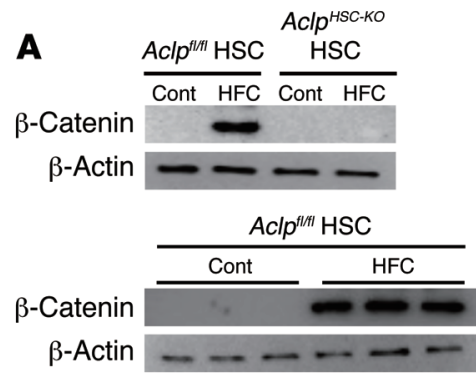

$\mathbf{B}$
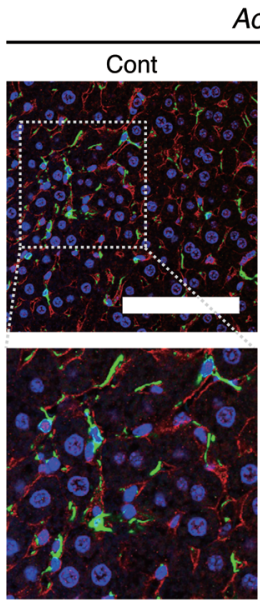

D
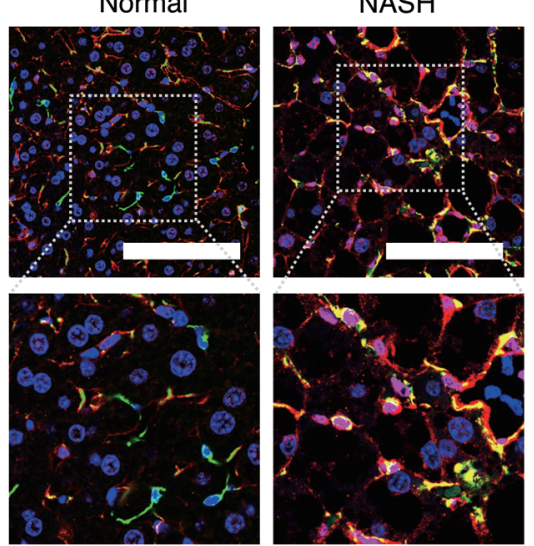

GFAP/ $\beta$-catenin/DAPI

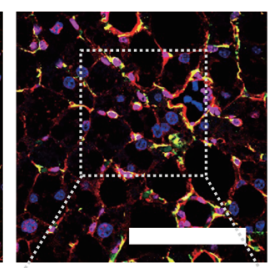

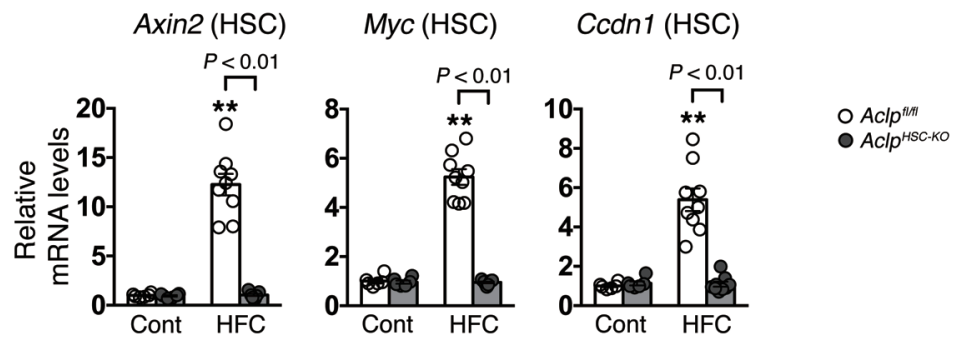

C

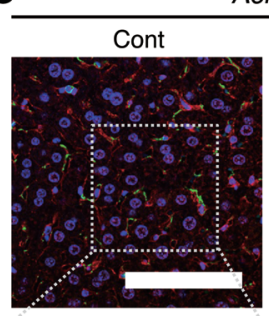

Aclp $p^{f / f t}$
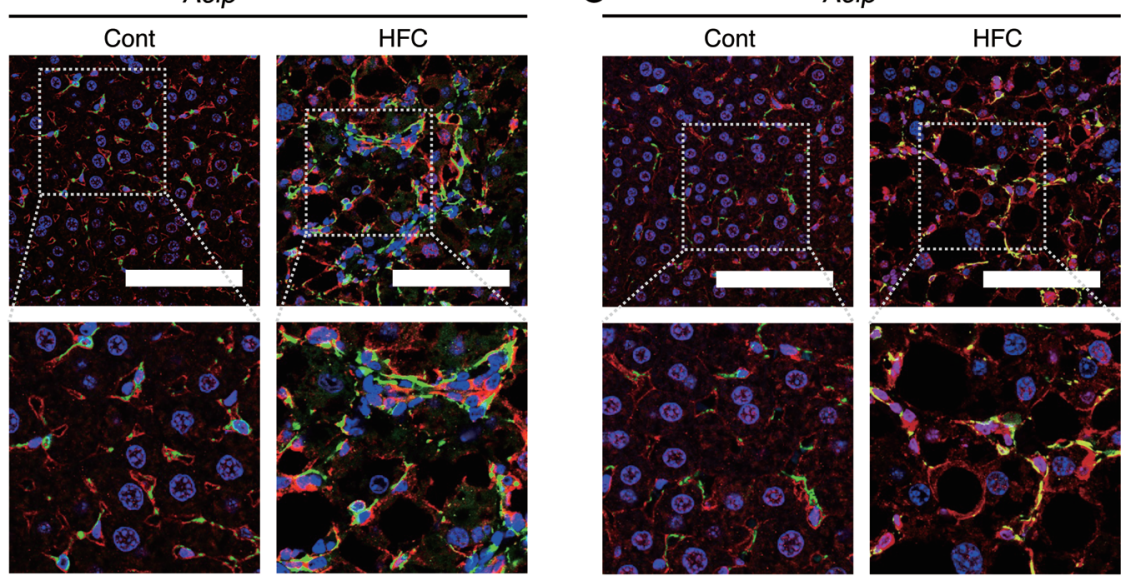

ACLP/ $\beta$-catenin/DAPI

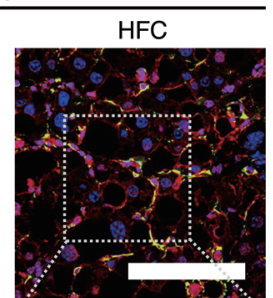

GFAP/ $\beta$-catenin/DAP

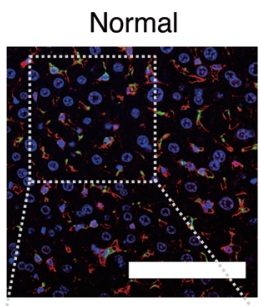

NASH
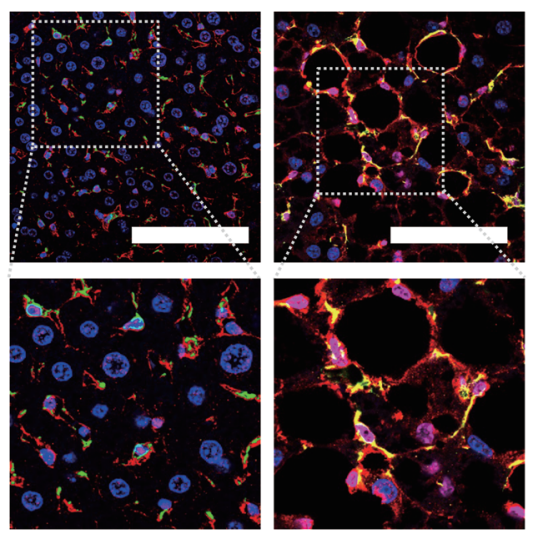

ACLP/ $\beta$-catenin/DAPI

Figure 3. ACLP expression in HSCs plays a major role in canonical WNT pathway activation in HSCs and in the resulting progression of liver fibrosis in human and murine NASH. (A-C) Eight-week-old male $A c / p^{f l / f l}$ and $A c / p^{H S C-K O}$ mice were fed a control diet ( $\left.n=6 / g r o u p\right)$ or an HFC diet ( $n=9 / \mathrm{group}$ ) for 24 weeks. (A) (Left panel) Western blot for $\beta$-catenin accumulation in HSCs in Aclp $p^{f / f l}$ mice and Aclp $p^{H S C-K O}$ mice. (Right panel) Quantification of $A x i n 2, M y c$, and Ccnd1 mRNA in HSCs in AcI $p^{f l / f l}$ mice and AcI ${ }^{H S C-K O}$ mice. ${ }^{* *} P<0.01$ compared with HSCs in Aclp ${ }^{f / / f l}$ mice fed a control diet. (B) Representative immunofluorescence double-staining images of $\beta$-catenin (red) and GFAP (green) in murine liver sections. Costained sites are shown in yellow. The nuclei were stained with DAPI (blue). $\beta$-Catenin/nucleus costained sites are shown in purple. Scale bars: $100 \mu \mathrm{m}$. Single-channel images are shown in Supplemental Figure 11. (C) Representative immunofluorescence double-staining images of $\beta$-catenin (red) and ACLP (green) in murine liver sections. Costained sites are shown in yellow. The nuclei were stained with DAPI (blue). $\beta$-Catenin/nucleus costained sites are shown in purple. Scale bars: $100 \mu \mathrm{m}$. Single-channel images are shown in Supplemental Figure 11. (D) Tissue samples from human control livers $(n=14)$ and NASH livers $(n=44)$ were used. (Left panel) Representative immunofluorescence double-staining images of $\beta$-catenin (red) and GFAP (green). (Right panel) Representative immunofluorescence double-staining images of $\beta$-catenin (red) and ACLP (green). Costained sites are shown in yellow, and nuclei were stained with DAPI (blue). $\beta$-Catenin/nucleus costained sites are shown in purple. Scale bars: $100 \mu \mathrm{m}$. Single-channel images are shown in Supplemental Figure 13. $P$ values obtained via 1-way ANOVA with Tukey's post hoc test. Data are shown as SEM. 
A
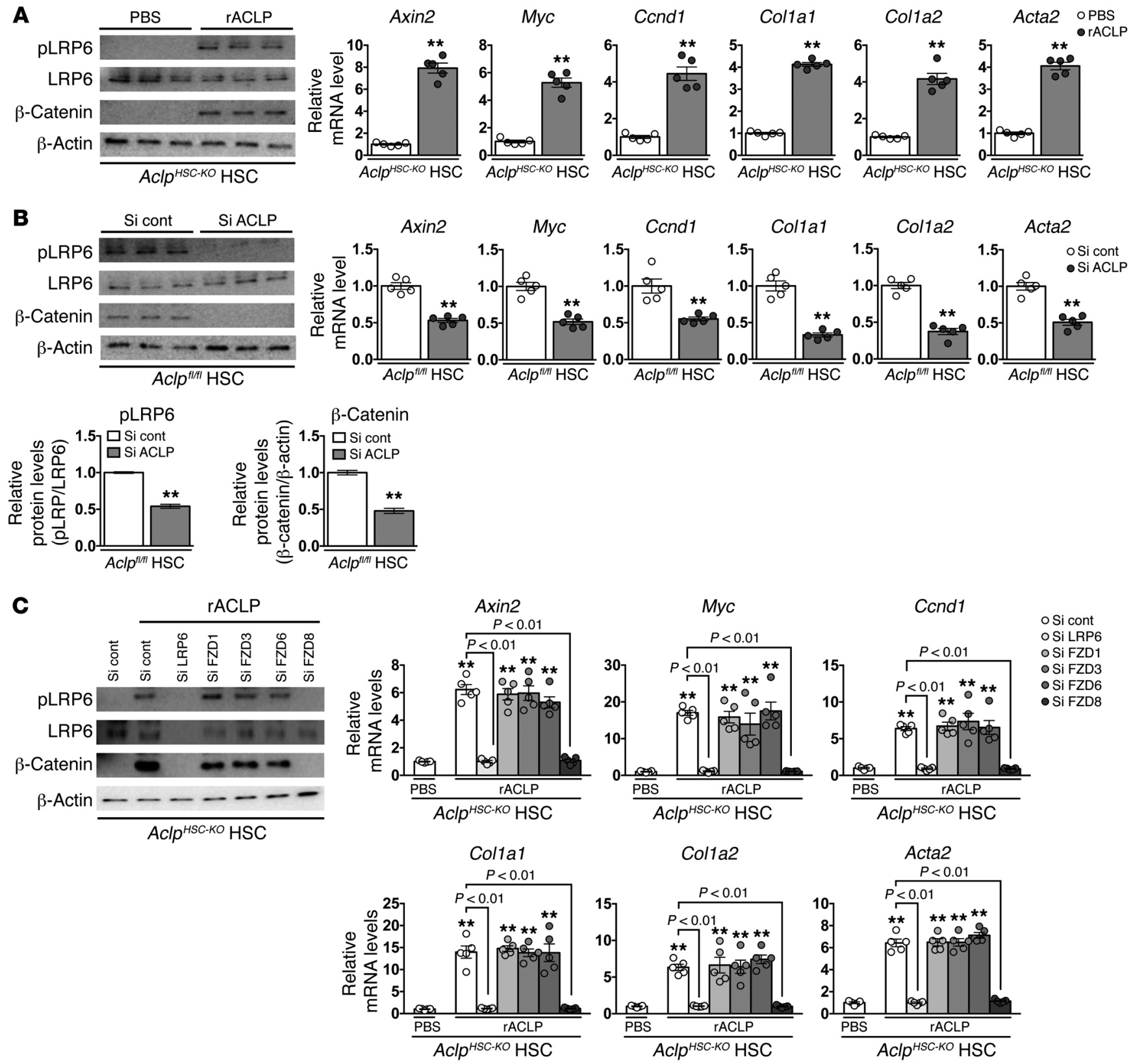

Figure 4. ACLP activates the canonical WNT pathway in HSCs in an FZD8/LRP6-dependent manner, thus promoting HSC activation. (A) (Left panel) Western blot for p-LRP6, LRP6, and $\beta$-catenin and (right panels) quantification of Axin2, Myc, Ccnd1, Col1a1, Col1 $\alpha 2$, and Acta2 mRNA in Aclp ${ }^{H S C-K O}$ HSCs treated with rACLP $(100 \mathrm{ng} / \mathrm{ml})$ or PBS for 12 hours ( $n=5 /$ group). ${ }^{* *} P<0.01 \mathrm{vs.} \mathrm{Acl}{ }^{H S C-K O}$ HSCs treated with PBS. (B) (Left panel) Western blot for and quantification of p-LRP6, LRP6, and $\beta$-catenin, and (right panels) quantification of Axin2, Myc, Ccnd1 Col1a1, Col1 $\alpha 2$, and Acta2 mRNA in Aclp fl/fl HSCs treated with ACLP or control siRNA for 72 hours ( $n=5 /$ group). ${ }^{* *} P<0.01 \mathrm{vs.} \mathrm{Acl} p^{f / f f l} \mathrm{HSC}$ s treated with control siRNA. (C) (Left panel) Western blot of p-LRP6, LRP6, and $\beta$-catenin, and (right panel) quantification of Axin2, Myc, Ccnd1 Col1a1, Col1 $\alpha 2$, and Acta2 mRNA in Aclp ${ }^{H S C-K O}$ HSCs treated with rACLP $(100 \mathrm{ng} / \mathrm{ml})$ for 12 hours in the presence of LRP6 siRNA, FZD1 siRNA, FZD3 siRNA, FZD6 siRNA, FZD8 siRNA, or control siRNA ( $n=5 /$ group). ${ }^{* *} P<0.01 \mathrm{vs.}$ AcI ${ }^{\text {HSC-KO }}$ HSCs treated with PBS in the presence of control siRNA. $P$ values obtained via 1-way ANOVA with Tukey's post hoc test (C), unpaired Student's $t$ tests (A and B, right panels), and Mann-Whitney $U$ test (B, left panel). Data are shown as SEM.

$D k k-1^{T g}$-derived HSCs (Supplemental Figure 12). Similarly, whereas the NASH model of Acl $p^{f l f l}$ mice demonstrated increased accumulation of $\beta$-catenin in HSCs, no such increase was observed in $A c l p^{H S C-K O}$ or $D k k-1^{T g}$ mice with NASH (Supplemental Figure 12).

Immunofluorescence double-staining of $\beta$-catenin and GFAP in human NASH liver specimens revealed increased $\beta$-catenin accumulation in the liver and, more specifically, in the HSCs (Fig- ure 3D and Supplemental Figure 13). Immunofluorescence doublestaining of ACLP and $\beta$-catenin in these specimens demonstrated a significant increase in ACLP/ $\beta$-catenin double-positive cells as compared with normal livers, and $\beta$-catenin was upregulated specifically in ACLP-positive cells in NASH (Figure 3D and Supplemental Figure 13). As for the mRNA expression levels of Wnt1, $W n t 3 a, W n t 5 a$, and $W n t 10 b$, which are reported to be correlated 
A

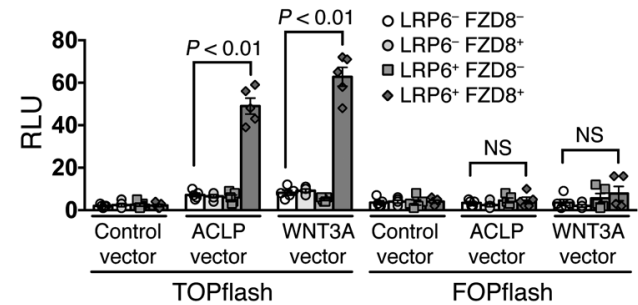

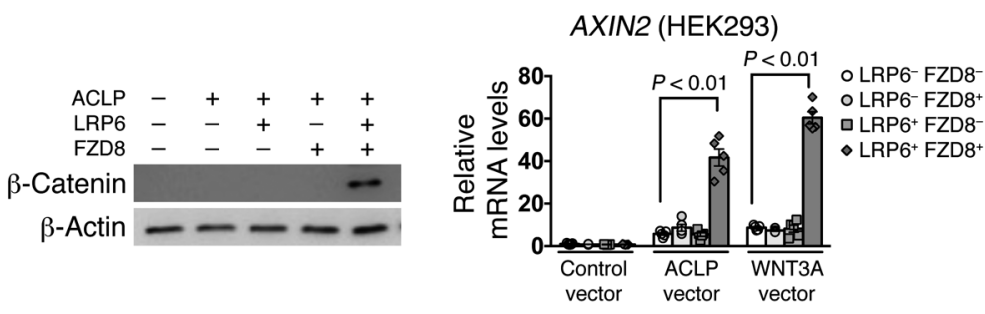

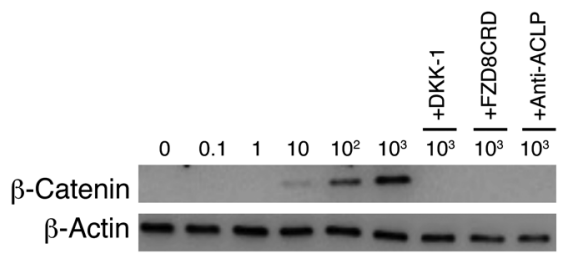

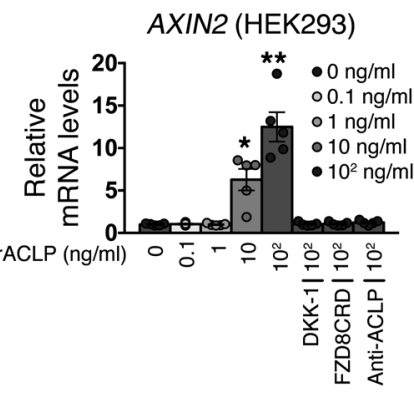

B

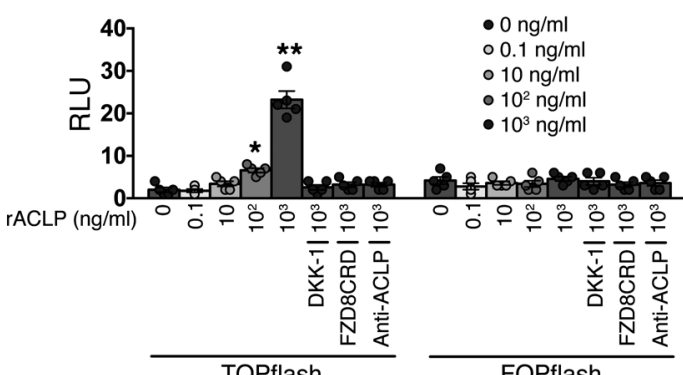

FOPflash

Figure 5. ACLP activates canonical WNT/ $\beta$-catenin signaling through FZD8 and LRP6. (A) Combinations of TOPflash and FOPflash luciferase reporter vectors, ACLP, WNT3A, LRP6, and FZD8 expression vectors, and control vector were transfected into HEK293 cells followed by culture for 36 hours ( $n=5 /$ group). (Left panel) Quantification of intracellular luciferase expression. (Middle panel) Western blot for $\beta$-catenin. (Right panel) Quantification of AXIN2 mRNA. (B) In addition to LRP6 and FZD8 expression vectors, TOPflash or FOPflash luciferase reporter vector was transfected into HEK293 cells. Following 24-hour culture, rACLP was administered at the indicated concentrations together with DKK-1 $(20 \mathrm{ng} / \mathrm{ml})$, FZD8CRD (20 $\mu \mathrm{g} / \mathrm{ml})$, and anti-ACLP antibody $(10 \mu \mathrm{g} / \mathrm{ml})(n=5 / g r o u p)$. After 12 hours of culturing, the cells were analyzed. (Left panel) Quantification of intracellular expression of luciferase. (Middle panel) Western blot for $\beta$-catenin. (Right panel) Quantification of $A X I N 2$ mRNA. ${ }^{*} P<0.05 ;{ }^{* *} P<0.01$ vs. control group (no rACLP). $P$ values obtained via 1-way ANOVA with Tukey's post hoc test. Data are shown as SEM.

with the canonical WNT pathway in liver pathology $(12,16,20$, 21), murine NAFLD livers and HSCs as well as human NAFLD liver samples demonstrated no changes when compared with their respective controls (Supplemental Figure 14). The above results indicated that in the progression of NASH, HSC-derived ACLP acts on HSCs themselves, thereby activating the HSCs and advancing liver fibrosis. Moreover, the canonical WNT pathway was indicated as being involved in NASH liver fibrosis progression downstream of ACLP signaling.

ACLP enhances canonical WNT signaling in HSCs and promotes HSC activation in an FZD8/LRP6-dependent manner. Next, we analyzed the involvement of ACLP in canonical WNT pathway activation in HSCs in more detail. In canonical WNT pathway activation, WNT binds to FZD family receptors and their coreceptors, LRP5 and LRP6, resulting in intracellular signal transmission (9). Therefore, we first examined the expression of FZD family receptors in murine HSCs. Murine HSCs primarily expressed FZD8, while expression of FZD1, FZD3, and FZD6 was low. Expression of FZD2, FZD4, FZD5, FZD7, FZD9, and FZD10 could not be detected (Supplemental Figures 15 and 16). Additionally, although the cells clearly expressed LRP6, LRP5 expression was hardly detectable (Supplemental Figure 15). Similarly, human HSCs expressed FZD8 and LRP6, while expression of FZD1, FZD3, FZD6, and LRP5 was very low and FZD2, FZD4, FZD5, FZD7, FZD9, and FZD10 expression could not be detected (Supplemental Figures 15 and 16).

We transfected a mouse ACLP overexpression vector into HEK293 cells and purified recombinant ACLP (rACLP) from the supernatant. rACLP purity was confirmed in nanoscale liquid chromatography/tandem mass spectometry (nano-LC-MS/MS) analyses (see Methods). Its band size indicated that rACLP was glycosylated, similar to native ACLP (13), which was confirmed by a deglycosylation-induced band shift (Supplemental Figure 17). To examine the direct effect of ACLP on HSCs, we administered rACLP to $A c l p^{H S C-K O}$-derived HSCs. Consequently, phosphorylation of LRP6 and accumulation of $\beta$-catenin were enhanced in these cells (Figure 4A). In addition, administration of rACLP significantly increased the mRNA levels of $A x i n 2, M y c$, and $C c n d 1$ as well as Col1a1, Col1a2, and Acta2 (Figure 4A). Administration of ACLP siRNA inhibited LRP6 phosphorylation and reduced $\beta$-catenin accumulation in HSCs derived from $A c l p^{\nexists f / f}$ mice as compared with administration of control siRNA (Figure 4B). In addition, ACLP siRNA administration significantly reduced $A x i n 2, M y c$, and Cond1 as well as Col1a1, Col1a2, and Acta 2 mRNA levels (Figure 4B). To determine whether the effects of ACLP on HSCs depend on FZD8/LRP6, we added rACLP following administration of FZD8 siRNA, FZD1 siRNA, FZD3 siRNA, FZD6 siRNA, LRP6 siRNA, or control siRNA to ACLP-deficient HSCs. The rACLPinduced enhancement of LRP6 phosphorylation, $\beta$-catenin accumulation, and Axin 2, Myc, Ccnd1 Col1a1, Col1 2 , and Acta2 mRNA expression was offset by preadministration of FZD8 siRNA and LRP6 siRNA, but not FZD1, FZD3, or FZD6 siRNA (Figure 4C). These results demonstrated that ACLP enhances canonical WNT signaling in HSCs and promotes HSC activation in an FZD8/ LRP6-dependent manner.

ACLP is a ligand of the FZD8 and LRP6 receptors. Because our results indicated that ACLP might act as a ligand in activating the canonical WNT pathway, we conducted a TOPflash/FOPflash assay in HEK293 cells. First, we prepared expression vectors har- 

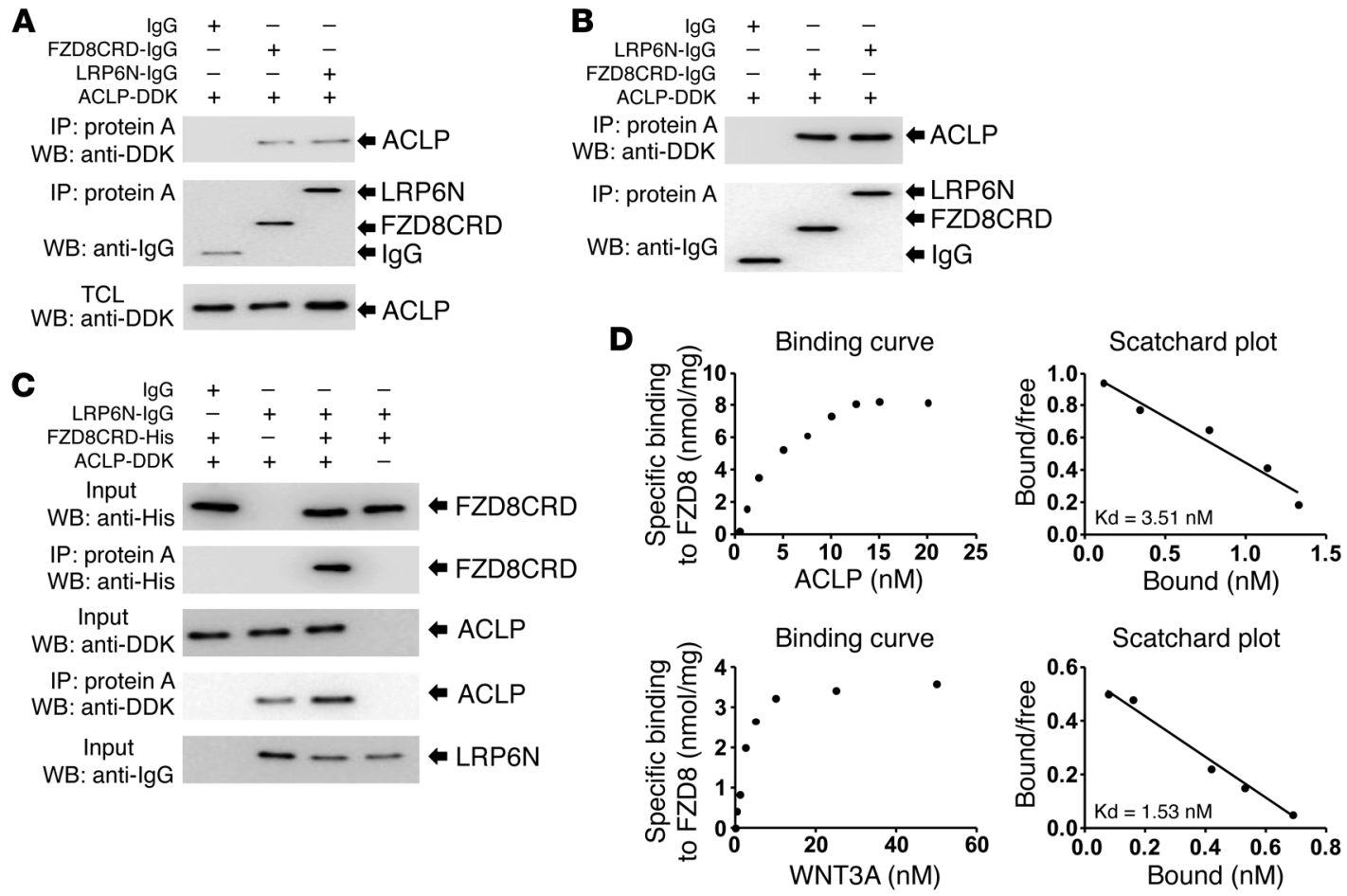

Figure 6. ACLP is a ligand that binds specifically to FZD8 and LRP6 receptors to form a ternary complex. (A) Combinations of ACLP-DDK and either human IgG, FZD8CRD-IgG, or LRP6N-IgG expression vectors (all $200 \mathrm{ng} /$ well) were transfected into HEK293 cells followed by culture for 72 hours. Cell lysates were used for immunoprecipitation. TCL, total cell lysates. (B) Combinations of rACLP-DDK and either FZD8CRD-IgG, LRP6N-IgG, or IgC were mixed to be used for immunoprecipitation. (C) Combinations of rACLP-DDK, FZD8CRD-His, LRP6N-IgG, and IgG were mixed to be used for immunoprecipitation. (D) Binding curve and Scatchard plot of binding between murine ACLP and murine FZD8/Fc (upper left panel and upper right panel, respectively); and binding curve and Scatchard plot of binding between murine WNT3A and murine FZD8/Fc (lower left panel and lower right panel, respectively). The experiments were repeated 3 times.

boring all possible combinations of FZD8 and LRP6, which were transfected into HEK293 cells. Then, we introduced the ACLP expression vector, a WNT3A expression vector, or a control vector, followed by TOPflash/FOPflash assay. When FZD8 or LRP6 was overexpressed solely, TOPflash reporter activity was very low, even when ACLP was subsequently expressed (Figure 5A). In contrast, when both FZD8 and LRP6 were expressed, subsequent expression of ACLP resulted in high reporter activity (Figure $5 \mathrm{~A})$. Western blotting revealed that ACLP expression markedly induced $\beta$-catenin accumulation only when both FZD8 and LRP6 were expressed in advance, which was consistent with the TOPflash/FOPflash assay results (Figure 5A). Consistently, ACLP expression significantly induced Axin 2 mRNA expression only under simultaneous expression of FZD8 and LRP6 (Figure 5A). Expression of WNT3A, used as a positive-control WNT-activating ligand, generated results identical to those observed for ACLP Tg expression (Figure 5A).

In HEK293 cells overexpressing both FZD8 and LRP6, we used the TOPflash/FOPflash assay upon addition of rACLP. The TOPflash reporter activity increased in a rACLP concentrationdependent manner, which was offset by the addition of DKK-1, the soluble WNT inhibitor FZD8CRD, or anti-ACLP antibody (Figure 5B). Similarly, intracellular $\beta$-catenin accumulation and Axin2 mRNA expression increased markedly in a rACLP concentrationdependent manner, which was abrogated upon addition of DKK-1, FZD8CRD, or anti-ACLP antibody (Figure 5B).
To examine the form of binding of ACLP to FZD8 and LRP6, we investigated whether ACLP binds to extracellular domains of FZD8 and LRP6. Expression vectors encoding human IgG fused to the extracellular domain of human LRP6 (LRP6N-IgG), human IgG fused to the cysteine-rich domain (CRD) of mouse FZD8 (FZD8CRD-IgG), and DDK-tagged mouse ACLP (ACLP-DDK) were cotransfected into HEK293 cells. Following immunoprecipitation of cell lysates, Western blotting revealed the existence of LRP6N-IgG/ACLP-DDK and FZD8CRD-IgG/ACLP-DDK immune complexes (Figure 6A). Next, to confirm binding in cell-free conditions, we expressed the mouse LRP6N-IgG and FZD8CRD-IgG constructs in HEK293 cells to prepare purified proteins (22). Mixtures of LRP6N-IgG and FZD8CRD-IgG with ACLP-DDK were analyzed by immunoprecipitation and Western blotting, which revealed the existence of LRP6N-IgG/ACLP-DDK and FZD8CRD-IgG/ACLP-DDK immune complexes (Figure 6B). Likewise, we prepared mouse FZD8CRD-His protein, which was mixed with LRP6N-IgG, ACLP-DDK, or both LRP6N-IgG and ACLP-DDK. Immunoprecipitation and Western blotting identified an immune complex of LRP6N-IgG, ACLP-DDK, and FZD8CRD-His (Figure 6C). Binding of LRP6N-IgG and FZD8CRD-His was not observed, demonstrating that these 2 proteins form a ternary complex through ACLP (Figure 6C).

Finally, to demonstrate that ACLP is a ligand of FZD8, we carried out a binding assay (23). The binding curve of ACLP and FZD8 was a concentration-dependent specific binding satura- 
A

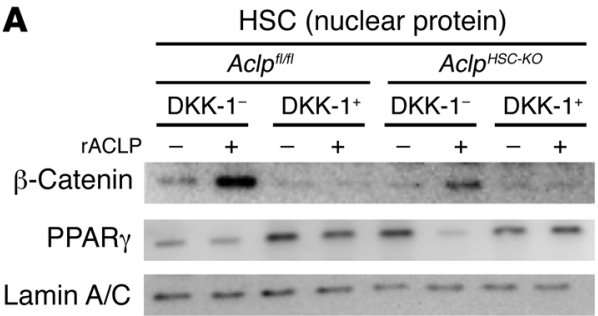

B PPAR $\gamma$ promoter activity (AC/ $\left.p^{H S C-K O} \mathrm{HSC}\right)$

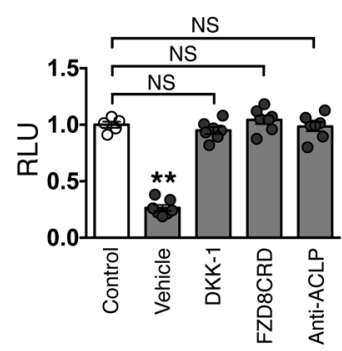

Pparg1 (ACIP ${ }^{\text {HSC-KO HSC) }}$

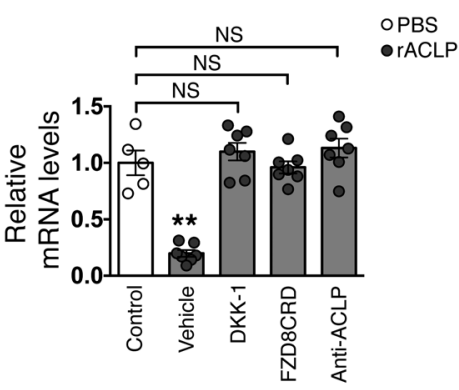

C

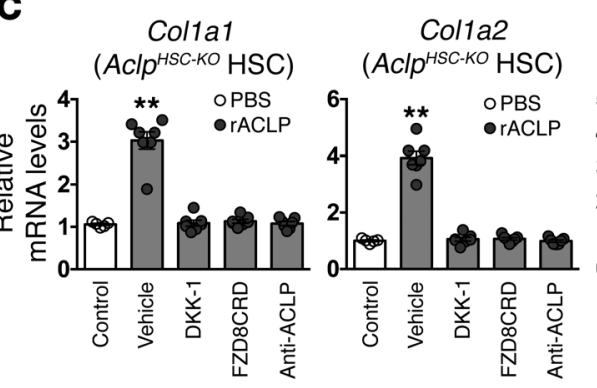

E
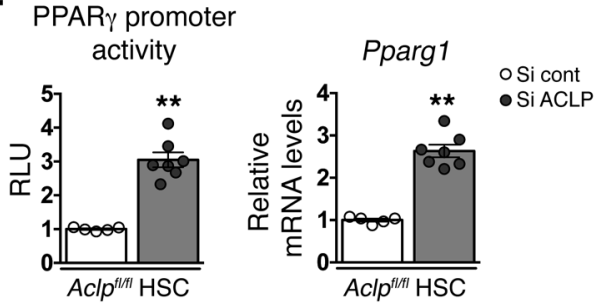

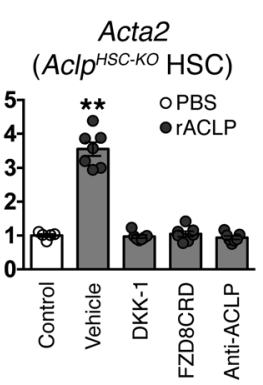

D
$\mathbf{F}$

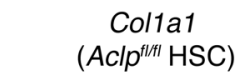

Col1a1 (Aclp ${ }^{H S C-K O}$ HSC)
Col1a2 (Aclp ${ }^{H S C-K O}$ HSC)
Acta2 (Aclp $\left.{ }^{H S C-K O} \mathrm{HSC}\right)$

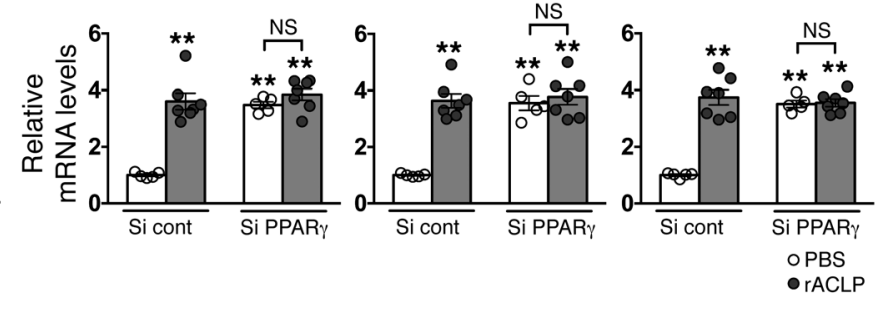

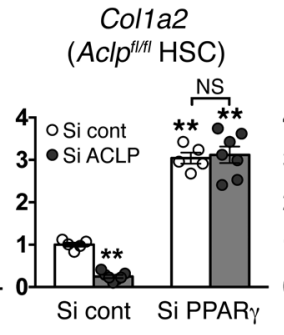

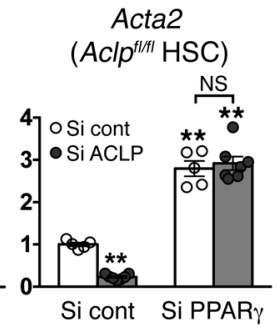

Figure 7. Activation of the canonical WNT pathway by ACLP activates HSCs through suppression of PPAR $\gamma$ signaling. (A) Western blot of nuclear $\beta$-catenin and PPAR $\gamma$ in AcI $p^{f / f l}$ and Aclp ${ }^{H S C-K O} \mathrm{HSCs}$ treated with rACLP $(100 \mathrm{ng} / \mathrm{ml})$ or PBS for 10 hours in the presence or absence of DKK-1 (20 $\left.\mathrm{ng} / \mathrm{ml}\right)$. Lamin A/C was used as a loading control for nuclear protein. The experiments were repeated 3 times. (B) (Left panel) PPAR $\gamma$ promoter activity and (right panel) quantification of Pparg1 mRNA expression in AclpHSC-KO HSCs treated with rACLP (100 ng/ml) ( $n=7 /$ group) or PBS ( $n=5 / \mathrm{group})$ for 12 hours in the presence or absence of DKK-1 $(20 \mathrm{ng} / \mathrm{ml})$, FZD8CRD $(20 \mu \mathrm{g} / \mathrm{ml})$, and anti-ACLP antibody $(10 \mu \mathrm{g} / \mathrm{ml})$. (C) Quantification of Col1a1, Col1a2, and Acta2 mRNA in Aclp ${ }^{\text {HSCKO }}$ HSCs treated with rACLP $(100 \mathrm{ng} / \mathrm{ml})(n=7 /$ group) or PBS ( $n=5 /$ group) for 10 hours in the presence or absence of DKK-1 (20 ng/ml), FZD8CRD $(20 \mu \mathrm{g} / \mathrm{ml})$, and anti-ACLP antibody $(10 \mu \mathrm{g} / \mathrm{ml}) .{ }^{* *} P<0.01 \mathrm{vs}$. Aclp ${ }^{H S C-K O} \mathrm{HSCs}$ treated with PBS. (D) Quantification of Col1a1, Col1 $\alpha 2$, and Acta2 mRNA in Aclp ${ }^{\text {HSCKO }}$ HSCs treated with rACLP $(100 \mathrm{ng} / \mathrm{ml})\left(n=7 /\right.$ group) or PBS $\left(n=5 /\right.$ group) for 12 hours in the presence of PPAR $\gamma$ siRNA or control siRNA. ${ }^{* *} P<0.01$ vs. AcIp ${ }^{H S C-K O}$ HSCs treated with PBS in the presence of control siRNA. (E) (Left panel) PPAR $\gamma$ promoter activity and (right panel) quantification of $P$ parg1 mRNA expression in Aclp $p^{f / f l}$ HSCs treated with ACLP siRNA ( $n=7 /$ group) or control siRNA ( $n=5 /$ group). (F) Quantification of Col1a1, Col1a2, and Acta2 mRNA in Aclp fl/fl HSCs treated with ACLP siRNA ( $n=7 /$ group) or control siRNA ( $n=5 /$ group) in the presence of PPAR $\gamma$ siRNA or control siRNA. ${ }^{* *} P<0.01$ vs. Acl $p^{f / f l}$ HSCs treated with control siRNA. $P$ values obtained via 1-way ANOVA with Tukey's post hoc test (B-D, and $\mathbf{F}$ ) and unpaired Student's $t$ tests (E). Data are shown as SEM.

tion curve (Figure 6D). Scatchard plot analysis demonstrated that ACLP binds to FZD8 with a high affinity equal to that of WNT3A $\left(\mathrm{Kd}_{\mathrm{ACLP}}: 3.51 \mathrm{nM}, \mathrm{Kd}_{\mathrm{WNT3A}}: 1.53 \mathrm{nM}\right.$; Figure 6D). Together, these results demonstrated that ACLP is a ligand unrelated to WNT proteins that binds specifically to FZD8 and LRP6, forming a ternary complex that activates WNT signaling.

ACLP activates HSCs via reduced PPAR signaling. Because ACLP/canonical WNT pathway activation triggered HSC activation, we examined this pathological mechanism further. It has been recently reported that activation of canonical WNT signaling represses PPAR $\gamma$ activity in HSCs, thereby promoting their activation $(12,16)$. Therefore, we examined PPAR $\gamma$ activity upon the addition of rACLP to HSCs. rACLP administration to ACLP-deficient HSCs increased nuclear $\beta$-catenin accumulation and reduced the nuclear PPAR $\gamma$ protein level (Figure 7A). When DKK-1 was administered concurrently, nuclear $\beta$-catenin accumulation did not increase, and the nuclear PPAR $\gamma$ protein level increased to that of the rACLP nonadministration group (Figure 7A). Next, we transfected an expression vector encoding PPAR $\gamma$ promoter-luciferase into ACLP-deficient HSCs and examined changes in PPAR $\gamma$ promoter activity resulting from rACLP addition. The addition of rACLP significantly reduced the PPAR $\gamma$ promoter activity and mRNA levels; however, concurrent administration of DKK-1, FZD8CRD, or anti-ACLP antibody recovered both the PPAR $\gamma$ promoter activity and mRNA expression to the control level (Figure 7B). mRNA expression of Col1a1, 

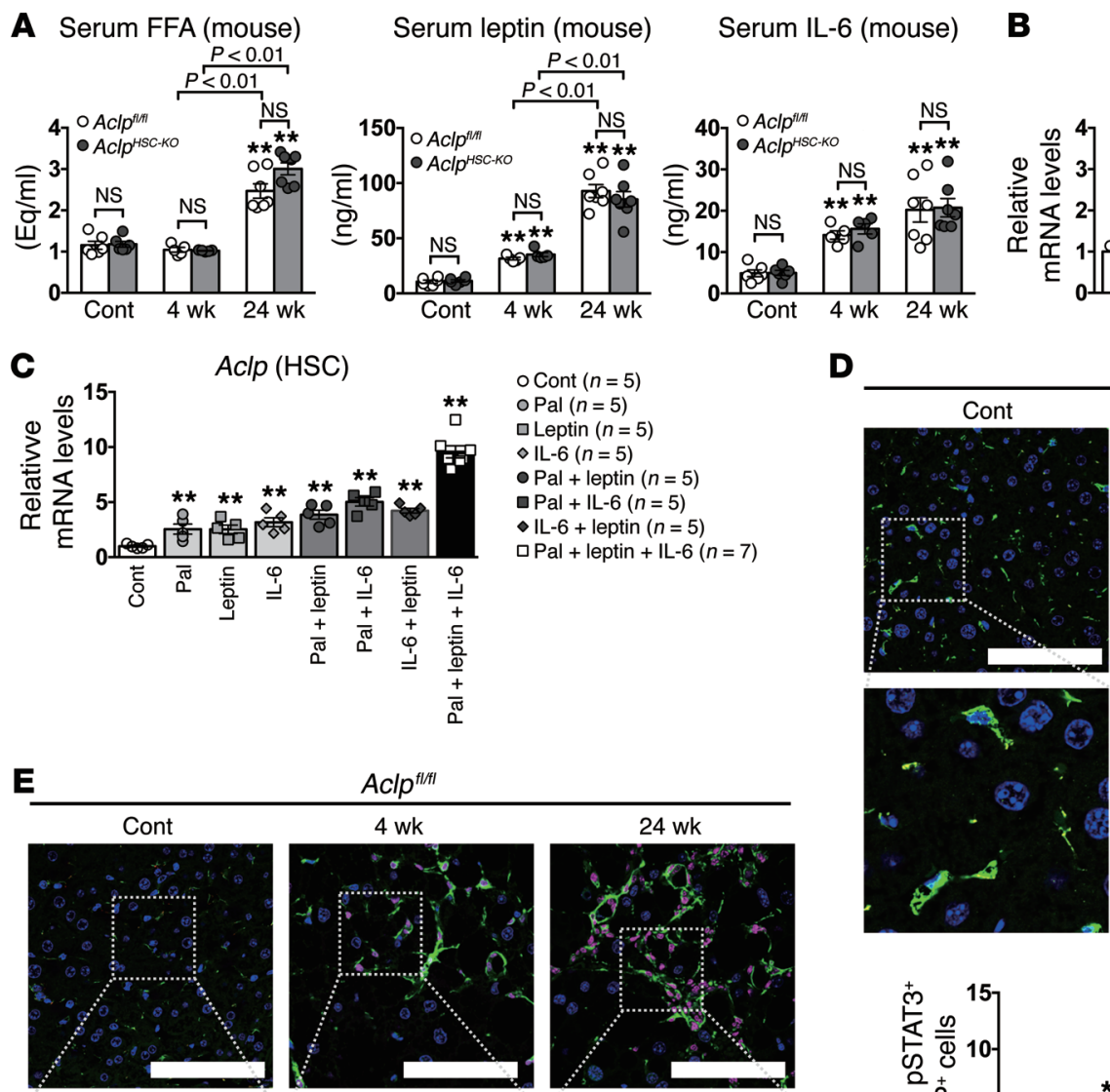

Aclp
(liver)

Aclp

Aclp

(liver)

(liver)

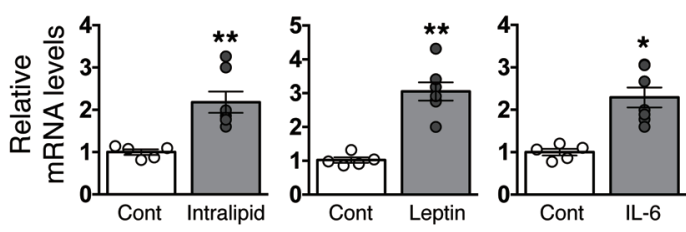

D

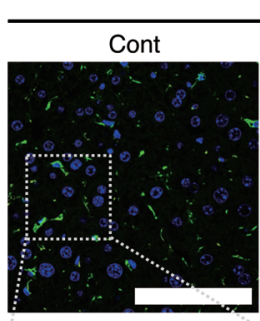

Aclp
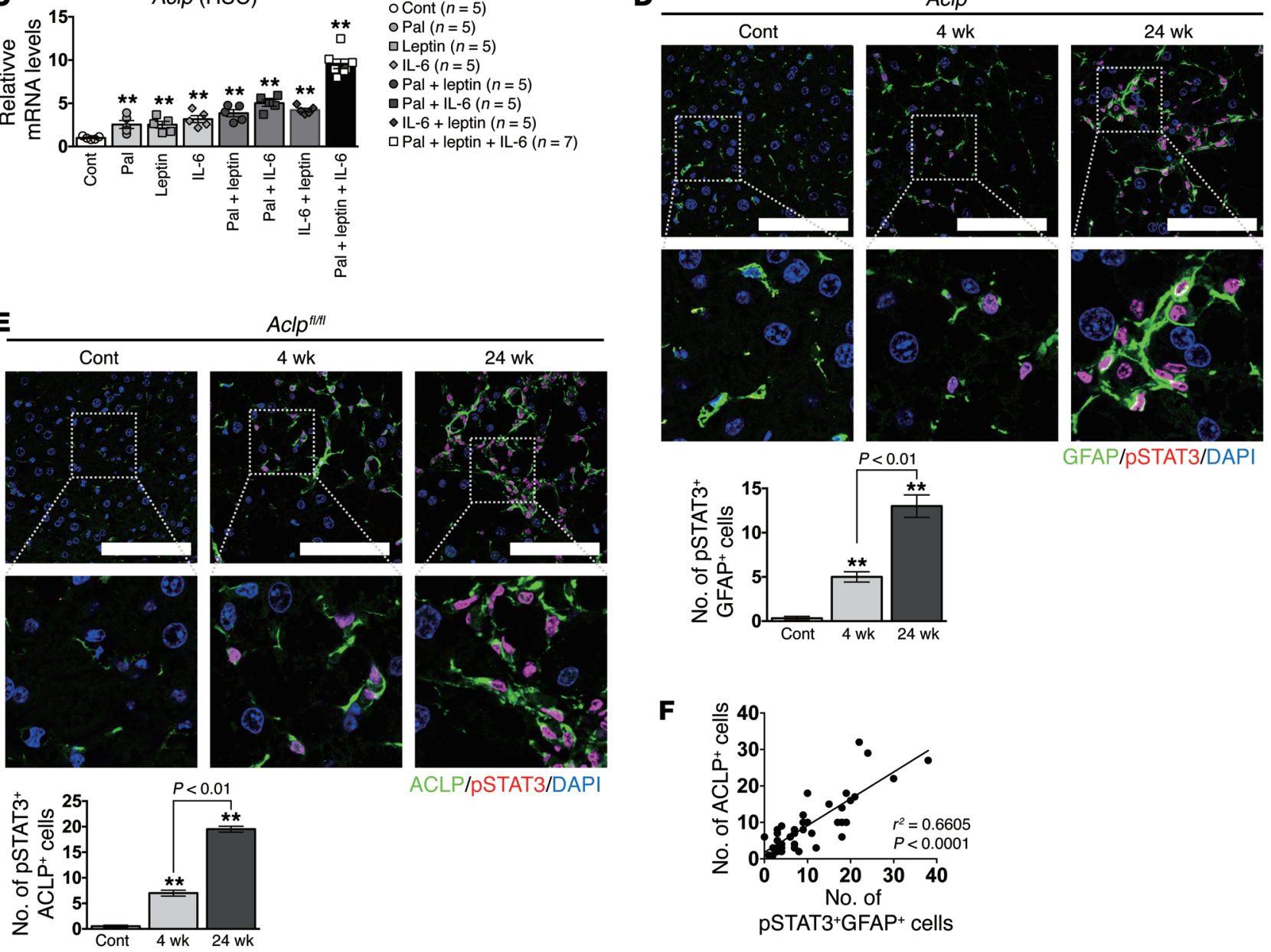

ACLP/pSTAT3/DAPI

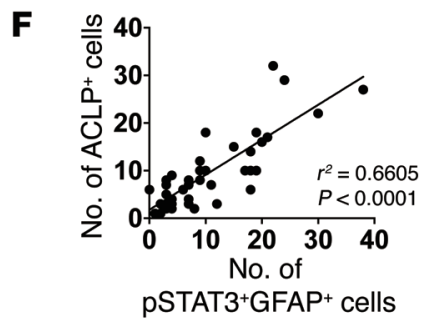

Figure 8. Obesity-related factors in serum enhance ACLP production in HSCs via activation of STAT3 signaling in murine NAFLD. (A, D-F) Eight-weekold male $A c / p^{f / f l}$ and $A c I p^{H S C-K O}$ mice were fed an HFC diet for 4 weeks ( $n=5 /$ group) or 24 weeks ( $n=7 /$ group) to prepare a murine model of NAFLD or were

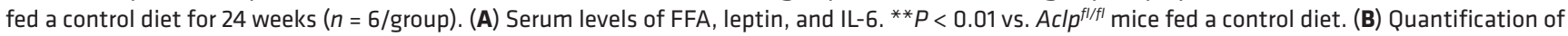
hepatic Aclp mRNA in 8-week-old WT mice 4 hours after treatment with intralipid ( $n=7 /$ group), leptin ( $n=7 /$ group), IL-6 ( $n=7 /$ group), or PBS ( $n=5$ / group). ${ }^{*} P<0.05$; ${ }^{*} P<0.01$ vs. control mice treated with PBS. (C) Quantification of Aclp mRNA in primary cultured murine HSCs treated with various combinations of palmitate $(200 \mu \mathrm{M})$, leptin $(100 \mathrm{ng} / \mathrm{ml})$, and IL-6 $(100 \mathrm{ng} / \mathrm{ml})$ for 6 hours $(n=5-7 /$ group $) .{ }^{* *} P<0.01$ vs. control HSCs treated with PBS. (D-F) Liver sections from control and NAFLD model Acl $p^{f / f l}$ mice were used. (D) (Upper panel) Representative images of immunofluorescence doublestaining for p-STAT3 (red) and GFAP (green). Scale bars: $100 \mu \mathrm{m}$. Single-channel images are shown in Supplemental Figure 20. (Lower panel) Quantification of pSTAT3/GFAP double-positive cells. The nuclei were stained with DAPI (blue). $p$-STAT3/nucleus costained sites are shown in purple. ${ }^{* *} P<0.01$ vs. Aclp $p^{f l / f l}$ mice fed a control diet. (E) (Upper panel) Representative immunofluorescence double-staining images of p-STAT3 (red) and ACLP (green). Scale bars: $100 \mu \mathrm{m}$. Single-channel images are shown in Supplemental Figure 21. (Lower panel) Quantification of pSTAT3/ACLP double-positive cells. The nuclei were stained with DAPI (blue). p-STAT3/nucleus costained sites are shown in purple. ${ }^{* *} P<0.01 \mathrm{vs}$. Acl $p^{f / f f}$ mice fed a control diet. (F) Correlation between p-STAT3/GFAP double-positive and ACLP-positive cells. $P$ values obtained via 1-way ANOVA with Tukey's post hoc test $(\mathbf{A}, \mathbf{C}, \mathbf{D}$, and E), unpaired Student's $t$ tests (B), and Pearson's correlation analysis (F). Data are shown as SEM.

Col1 $\alpha 2$, and Acta2 was significantly increased by the addition of rACLP, while concurrent administration of DKK-1, FZD8CRD, or anti-ACLP antibody reversed it to the control level (Figure 7C). Administration of PPAR $\gamma$ siRNA to ACLP-deficient HSCs result- ed in HSC activation and significantly increased expression of Col1a1, Col1a2, and Acta2 mRNA. Moreover, in this condition, the addition of ACLP did not yield any changes in the mRNA levels of these molecules (Figure 7D). 
A

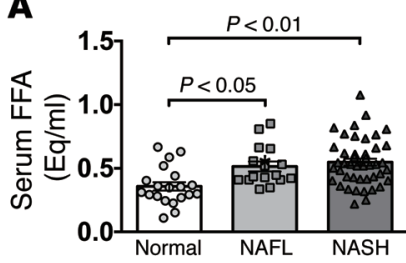

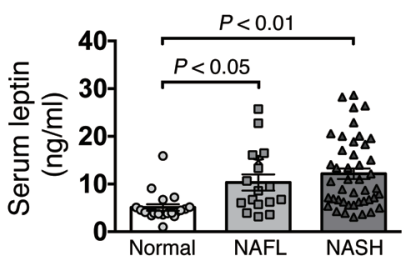

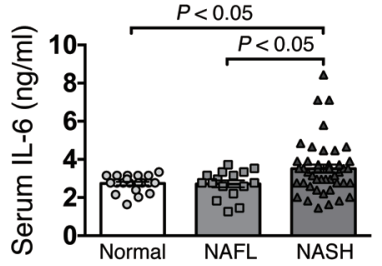

D

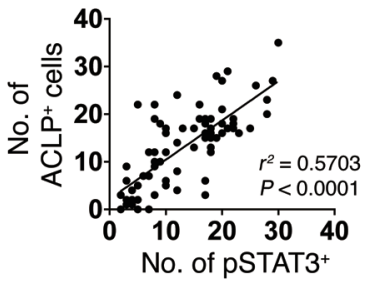

$\mathrm{GFAP}^{+}$cells

B
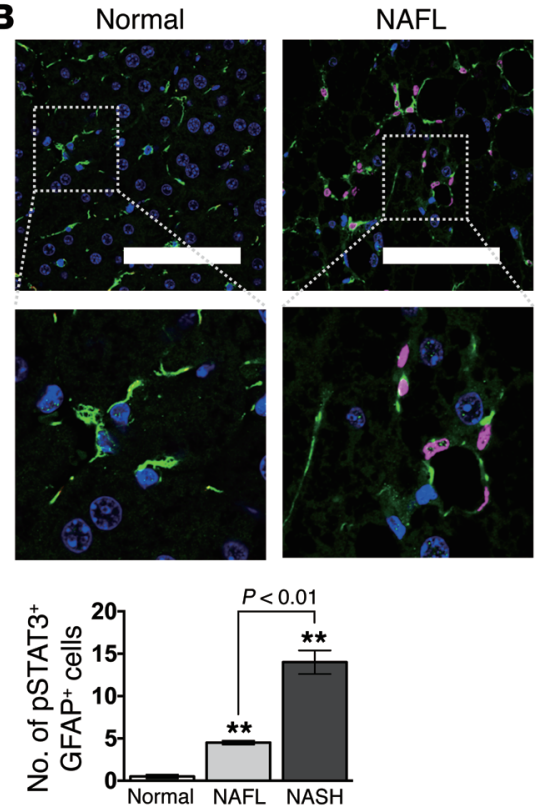
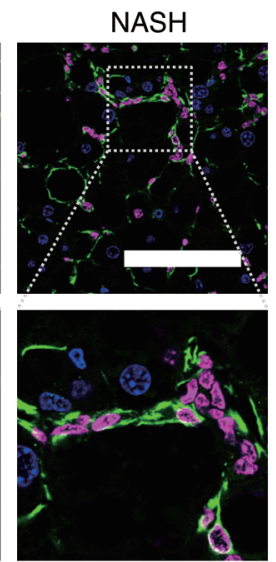

C
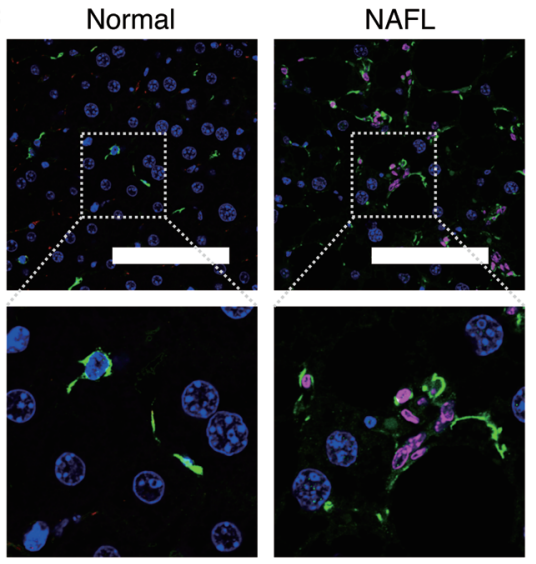

GFAP/PSTAT3/DAPI
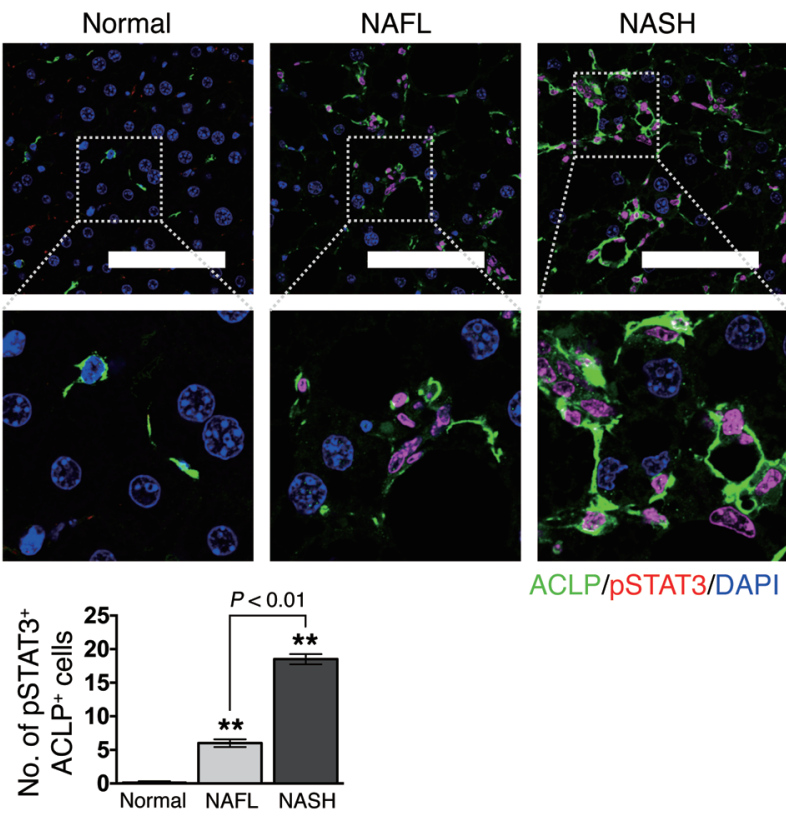

ACLP/PSTAT3/DAPI

Figure 9. Obesity-related factors in serum enhance ACLP production in HSCs via activation of STAT3 signaling in the livers of NAFLD patients. (A) Serum levels of FFA, leptin, and IL-6 in healthy control subjects $(n=20)$, NAFL patients $(n=16)$, and NASH patients $(n=44)$. (B-D) Liver sections from controls ( $n=14)$, NAFL patients $(n=16)$, and NASH patients $(n=44)$ were used. (B) (Upper panel) Representative images of immunofluorescence double-staining of p-STAT3 (red) and GFAP (green). Scale bars: $100 \mu \mathrm{m}$. Single-channel images are shown in Supplemental Figure 23. (Lower panel) Quantification of pSTAT3/GFAP double-positive cells. The nuclei were stained with DAPI (blue). p-STAT3/nucleus costained sites are shown in purple. ${ }^{* *} P<0.01$ vs. control subjects. (C) (Upper panel) Representative immunofluorescence double-staining images of p-STAT3 (red) and ACLP (green). Scale bars: 100 $\mu \mathrm{m}$. Single-channel images are shown in Supplemental Figure 23. (Lower panel) Quantification of pSTAT3/ACLP double-positive cells. The nuclei were stained with DAPI (blue). p-STAT3/nucleus costained sites are shown in purple. ${ }^{* *} P<0.01$ vs. control liver samples. (D) Correlation between p-STAT3/GFAP double-positive and ACLP-positive cells. $P$ values obtained via 1-way ANOVA with Tukey's post hoc test (A-C) and Pearson's correlation analysis (D). Data are shown as SEM.

Next, we transfected a PPAR $\gamma$ promoter-luciferase vector into $A c l p^{f / f l}$ HSCs to examine changes in PPAR $\gamma$ promoter activity induced by the addition of ACLP siRNA. While the administration of ACLP siRNA inhibited endogenous ACLP production, it significantly enhanced PPAR $\gamma$ promoter activity and the Pparg1 mRNA level (Figure 7E). ACLP silencing inhibited HSC activation and significantly reduced mRNA expression of Col1a1, Col1a2, and Acta2 (Figure 7F). Administration of PPAR $\gamma$ siRNA to Aclpt/fl HSCs activated the HSCs and significantly increased Colla1, Col1a2, and Acta2 mRNA, while the addition of ACLP siRNA to these cells had no effect (Figure 7F). These results demonstrated that ACLP activates the canonical WNT pathway in HSCs, thus reducing PPAR $\gamma$ signaling by reducing PPAR $\gamma$ promoter activity, consequently activating HSCs.

In NAFLD, obesity-related factors in serum enhance ACLP expression in HSCs through the activation of STAT3 signaling. As hepatic expression of ACLP increased with NAFLD progres- sion in both humans and mice, we examined the mechanism of ACLP expression. In the murine model of NAFLD, we found that an HFC diet increased serum free fatty acid (FFA), leptin, and IL-6 levels over time, while $A c l p^{H S C-K O}$ and Aclp $p^{A f l}$ mice did not differ from each other in these aspects (Figure 8A). Administration of intralipid, leptin, or IL-6 to WT mice significantly increased the hepatic Aclp mRNA levels, specifically in HSCs (Figures 8B and Supplemental Figure 18). Palmitate, leptin, and IL-6, which are known to activate intracellular STAT3 signaling (24-26), increased ACLP expression additively and enhanced the phosphorylated STAT3 (p-STAT3) level in primary cultured murine HSCs (Figure 8C and Supplemental Figure 19). Concurrent administration of STAT3 inhibitor reduced Aclp mRNA to the level observed in the control group (Supplemental Figure 19). In vivo administration of intralipid, leptin, or IL-6 to WT mice also resulted in significantly elevated p-STAT3 levels in HSCs (Supplemental Figure 18). Immunofluorescence double-staining for 
ACLP and p-STAT3 revealed that the number of ACLP/p-STAT3 double-positive cells increased significantly with the administration of intralipid, leptin, or IL-6 (Supplemental Figure 18).

Furthermore, p-STAT3 increased in HSCs of the murine NAFLD model over time (Figure 8D and Supplemental Figure 20), and the number of ACLP/p-STAT3 double-positive cells increased significantly as NAFLD progressed (Figure 8E and Supplemental Figure 21). Plotting of the numbers of p-STAT3/GFAP double-positive and ACLP-positive cells in a set area of NAFLD liver revealed a significant correlation between the numbers of these 2 cell types (Figure 8F).

Next, we conducted a similar examination of human NAFLD liver specimens. NAFLD patients demonstrated elevated serum concentrations of FFA, leptin, and IL-6 as NAFLD progressed (Figure 9A). When palmitate, leptin, or IL-6 was added to primary cultured HSCs isolated from normal human livers, as in mice, p-STAT3 increased significantly (Supplemental Figure 22). While ACLP mRNA expression in the HSCs increased significantly following the addition of palmitate, leptin, or IL-6, upon concurrent administration of STAT3 inhibitor, ACLP mRNA remained at the level of the control (Supplemental Figure 22). Consistently, p-STAT3 levels in HSCs in NAFLD liver sections and the number of ACLP/p-STAT3 double-positive cells significantly increased as NAFLD progressed (Figure 9, B and C, and Supplemental Figure 23). Furthermore, we found a significant correlation between the numbers of p-STAT3/ GFAP double-positive cells and ACLP-positive cells (Figure 9D).

Based on these findings, we propose the following mechanism for pathogenesis of NAFLD: serum concentrations of obesityrelated factors, such as FFA, leptin, and IL-6 are elevated, and these obesity-related factors act on HSCs and activate STAT3 signaling, thereby increasing ACLP expression. We also found that ACLP preceded the upregulation of fibrogenic genes during HSC activation in NAFLD (Figure 1, Figure 2, A and B, and Supplemental Figure 24).

Vitamin A-coupled liposomes have been reported to be internalized specifically by activated HSCs (27). Finally, considering clinical application, we examined whether inhibition of ACLP in activated HSCs, by administering vitamin A-coupled liposomes bearing ACLP siRNA (VA-lip-siRNA-ACLP), could reverse liver fibrosis in the mouse NASH model with established fibrosis. WT mice fed an HFC diet for 24 weeks were administered VA-lipsiRNA-ACLP or VA-lip-siRNA-control (Supplemental Figure 25). Activated HSCs reverted to more quiescent HSCs after taking up VA-lip-siRNA-ACLP, and as a result of this increase, liver fibrosis in NASH was improved (Supplemental Figure 25). In contrast, no significant differences in the severity of liver fibrosis were observed between $A c l p^{H S C-K O}$ and $A c l p^{f / f l}$ mice in other liver fibrosis models, such as the carbon tetrachloride $\left(\mathrm{CCl}_{4}\right)$ model and the bile duct ligation (BDL) model (Supplemental Figures 26 and 27), suggesting more predominant roles of other WNT ligands, such as WNT1, WNT3, and WNT10b, in these fibrotic conditions.

\section{Discussion}

In the present study, we demonstrated that ACLP, a protein secreted specifically by HSCs in the liver, is a ligand that activates the canonical WNT pathway via specific binding to FZD8 and LRP6. Both WNT and ACLP are secreted glycoproteins with affinity for $\operatorname{ECM}(14,28)$.
Without WNT stimulation, $\beta$-catenin in the cytoplasm binds to a "destruction complex" composed of AXIN, adenomatous polyposis coli, and glycogen synthase kinase-3 and is phosphorylated, leading to its ubiquitination and degradation by proteasomes $(9,29)$. In the canonical WNT pathway, WNT forms a complex with the cell-surface FZD receptor and the LRP5/6 receptor, a single-pass transmembrane molecule. FZD proteins are 7-transmembrane receptors that possess extracellular N-terminal CRDs to which WNT binds. Complex formation of WNT and its receptors induces the association of AXIN with p-LRP, destruction of the complex, and stabilization of $\beta$-catenin, which then accumulates in the cytoplasm. Subsequently, $\beta$-catenin translocates to the nucleus where it interacts with the transcription factor $\mathrm{T}$ cell factor (TCF)/lymphoid enhancer factor (LEF) to promote AXIN2, cMYC, and cyclin D1 transcription $(9,29)$. The secreted protein Norrin, a non-WNT-family WNT ligand, activates the canonical WNT pathway by binding to FZD4CRD (30). Another mechanism of canonical WNT signaling activation involves the cleavage of LRP6 by complement C1q (31).

We demonstrated that in humans and mice, ACLP expression in HSCs markedly increased in NASH, concomitant with the activation of canonical WNT signaling. Moreover, enhancement of canonical WNT signaling in the NASH liver occurred almost exclusively in HSCs. In HSC-specific ACLP-KO mice, canonical WNT signaling in HSCs in NASH was inhibited to the level observed in control mice fed a normal diet. DKK-1 inhibits canonical WNT signaling either by binding to LRP6 receptors or by inhibiting the binding of WNT to LRP6, removing LRP6 from the lipid raft, internalizing it in a clathrin-dependent manner, and removing it from the cell membrane $(17,18)$. Therefore, we prepared $A c l p^{H S C-K O} D k k-1^{T g}$ mice, allowing in vivo examination of ACLP function related to canonical WNT signaling. In DKK-1 Tg NASH model mice, canonical WNT signaling was inhibited to the control level and was not affected by ACLP expression in HSCs, indicating that ACLP is involved in the canonical WNT pathway in HSCs. These results also suggested that in NASH, ACLP plays an important role in canonical WNT pathway activation in HSCs.

Furthermore, our in vitro and in vivo examinations demonstrated that ACLP, similar to classical ligands of the canonical WNT pathway, binds specifically to FZD8CRD and the extracellular domain of LRP6 to form a ternary complex, triggering $\beta$-catenin-dependent gene activation through LRP6 phosphorylation. In addition, the binding affinity of ACLP for FZD8 was as high as that of WNT3A.

WNT proteins are extremely hydrophobic and adhere strongly to the cell membrane and ECM, where they act on the secreting cell itself or on its surroundings $(28,29)$. ACLP is known as an ECM protein; in NASH, ACLP produced specifically in the HSCs activated canonical WNT signaling in the HSCs themselves.

The canonical WNT pathway inhibits preadipocyte differentiation into adipocytes by inhibiting PPAR $\gamma$ activity (32). Myoblasts, which, like preadipocytes, are mesodermal cells, have been demonstrated to differentiate into adipocytes as a result of canonical WNT pathway inhibition (32). Recent lineage-tracing studies in mice have shown that HSCs differentiate from mesodermal cells within the septum transversum (33). Quiescent HSCs store lipid droplets, whereas transdifferentiated (activated) HSCs deplete lipid droplets and reduce the expression of PPAR $\gamma$ 
and other adipogenic genes $(12,34)$. Therefore, HSC differentiation, of which PPAR $\gamma$ signaling is the master regulator, is considered analogous to dedifferentiation from adipocytes to preadipocytes $(12,34)$. It has in fact been recently reported that when the canonical WNT pathway is activated, methyl-CpG binding protein 2 and $\mathrm{H} 3 \mathrm{~K} 27$ dimethylation trigger epigenetic repression of PPAR $\gamma$ expression, resulting in HSC activation (16). As a ligand of the canonical WNT pathway, ACLP reduced PPAR $\gamma$ promoter activity in HSCs via the activation of canonical WNT signaling, thus weakening PPAR $\gamma$ signaling, which in turn resulted in HSC activation. We consider this to be the main pathological mechanism of ACLP in the progression of NASH.

NAFLD, regarded as a hepatic manifestation of metabolic syndrome, is often accompanied by obesity and metabolic syndrome as background factors, indicating that these factors are involved in NAFLD. In obesity, there is an increased supply of FFA from adipose tissues and diet to the liver (35). In fatty liver, a large share of TG is known to originate from serum FFAs (36). Furthermore, leptin and IL- 6 are typical proinflammatory adipocytokines, serum concentrations of which are elevated in obesity (37). In our investigations of murine models and humans, serum concentrations of obesity-related factors, such as FFA, leptin, and IL-6, increased as NAFLD progressed. Leptin and IL- 6 are known to activate STAT3 in HSCs $(24,25)$, while FFA is also reported to activate STAT3 signaling (26). Our in vivo and in vitro analyses of murine models and human samples demonstrated that FFA, leptin, and IL-6, the serum levels of which are elevated in NAFLD, additively enhance ACLP production in HSCs through STAT3 activation.

In the future, further studies demonstrating that ACLP overexpression in HSCs triggers spontaneous HSC activation and liver fibrosis in vivo could prove that ACLP is sufficient for in vivo HSC activation. On the other hand, it has been reported that ACLP secreted by lung fibroblasts was involved in lung fibrosis by promoting the fibroblast-to-myofibroblast transition partly through the activation of TGF- $\beta$ signaling $(38,39)$. Unlike ACLP in the lung, ACLP in the liver is secreted specifically by HSCs and not fibroblasts; this could explain the differences in the mechanisms of action of ACLP in an autocrine manner between that in the liver and that in the lung.

In summary, we demonstrated that ACLP, a glycosylated, secreted protein, which in the liver is specifically expressed by HSCs, is a ligand unrelated to WNT proteins that binds specifically to FZD8 and LRP6 to form a ternary complex and activates the canonical WNT pathway. FFA, leptin, and IL-6, whose serum concentrations increase in association with obesity, a background factor of NAFLD, enhance ACLP production in HSCs. Further, we demonstrated that ACLP attenuates PPAR $\gamma$ signaling via the activation of the canonical WNT pathway in HSCs, thus promoting HSC activation and exacerbating the progression of liver fibrosis in NASH. Enhancement of canonical WNT signaling in the liver in NASH was almost completely localized in HSCs; ACLP, as a WNT ligand, was demonstrated to play a major role in this enhanced activation (Supplemental Figure 28). These results suggest that the activation of the canonical WNT pathway by ACLP in HSCs is a potential target for the diagnosis and treatment of NASH. Future studies should conduct a detailed examination of the potential clinical applications of ACLP.

\section{Methods}

Please refer to the Supplemental Methods section for more detailed descriptions.

Antibodies, expression vectors, and reagents. Anti-ACLP and antip-STAT3 (Tyr705) antibodies were purchased from Santa Cruz Biotechnology Inc. Anti- $\beta$-actin, anti-LRP6, anti-LRP5, and anti-p-LRP6 (Ser1490) antibodies were purchased from Cell Signaling Technology (Danvers). Anti- $\alpha \mathrm{SMA}$, anti-desmin, anti- $\beta$-catenin, anti-lamin A/C, anti-FZD3, anti-FZD5, anti-FZD6, anti-FZD7, anti-FZD8, anti-FZD10, anti-IgG, anti-p-STAT3 (Tyr705) (EP2147Y), and anti-PPAR $\gamma$ antibodies were purchased from Abcam. Anti-GFAP antibodies were purchased from DAKO and Abcam. Anti-FZD1 antibody was purchased from R\&D Systems. Anti-FZD4 antibody was purchased from Thermo Fisher Scientific. Anti-CRE recombinase antibody was purchased from Merck Millipore. Anti-F4/80 antibody was purchased from AbD Serotec. Anti-FLAG M2 monoclonal antibody (anti-DDK; F1804) and anti-FZD2 antibody were purchased from Sigma-Aldrich. Anti-rabbit IgG HRP-linked antibody was purchased from GE Healthcare. AntiHis tag antibody was purchased from Medical \& Biological Laboratories. See Supplemental Table 1 for information about the antibodies.

Mouse ACLP-DDK expression vector was purchased from OriGene Technologies. TOPflash and FOPflash luciferase reporters and expression vectors for human LRP6, human LRP6N-IgG, mouse FZD8, mouse FZD8CRD-IgG, and mouse WNT3A were purchased from Addgene. In order to prepare a mouse FZD8CRD-His expression vector, the KOD-Plus-Mutagenesis Kit (Toyobo Life Science) was used to introduce a $6 \mathrm{xHis}$ tag and a stop codon immediately after the FZD$8 \mathrm{CRD}$ sequence in the mouse FZD8CRD expression vector. Human IgG expression vector was purchased from Sigma-Aldrich.

Recombinant mouse DKK-1, mouse leptin, mouse IL-6, and human FZD8/Fc were purchased from R\&D Systems. Recombinant human FZD1 was purchased from Thermo Fisher Scientific. Recombinant human FZD2, human FZD4, human FZD5, human FZD6, human FZD7, and human FZD10 protein were purchased from Abnova. Recombinant human FZD3 protein was purchased from Abcam. Recombinant human FZD8 protein was purchased from R\&D Systems.

Intralipid and palmitate were purchased from Sigma-Aldrich. Stattic was purchased from Merck Millipore. ACLP siRNA, PPAR $\gamma$ siRNA, LRP6 siRNA, FZD3 siRNA, FZD6 siRNA, FZD8 siRNA, and control siRNA were purchased from Thermo Fisher Scientific. Tris$\mathrm{HCl}$, pyruvic acid, HEPES, EDTA, EGTA, and CHAPS were purchased from Sigma-Aldrich. $\mathrm{NaCl}$, mannitol, and sucrose were purchased from Wako Pure Chemical Industries. Spermine and spermidine were purchased from Tokyo Chemical Industry.

Animal studies. As murine models of NAFLD, 8-week-old male C57BL/6J mice (CLEA Japan), Aclp $p^{f / f l}$ mice, Aclp ${ }^{H S C-K O}$ mice, Aclp $p^{f / f l}$ $D k k-1^{T g}$ mice, or $A c l p^{H S C-K O} D k k-1^{T g}$ mice were fed a CE-2 (CLEA Japan) or HFC diet (prepared by CLEA Japan: high fat diet [101447: Dyets] with $1 \%$ cholesterol), as previously described (15), for 4 weeks or 24 weeks. For GTT at 21 weeks of control or HFC diet, the mice were fasted for 16 hours and were then intraperitoneally administered $1.5 \mathrm{~g} / \mathrm{kg}$ body weight of glucose. For ITT at 22 weeks, the mice were fasted for 4 hours and were then intraperitoneally administered $0.5 \mathrm{U} / \mathrm{kg}$ body weight of insulin. For PTT at 23 weeks, the mice were fasted for 16 hours and were then intraperitoneally administered pyruvate $(2 \mathrm{~g} / \mathrm{kg}$ body weight) dissolved in PBS. Blood glucose was measured at 0,15 , 30,60 , and 90 minutes after administration using LabAssay Glucose 
(Wako Pure Chemical Industries) and a microplate reader (Bio-Rad). In an acute administration experiment, 8-week-old male C57BL/6J mice, which had been fasted for 12 hours, were given an intraperitoneal injection of recombinant mouse leptin ( $5 \mathrm{mg} / \mathrm{g}$ body weight) and a tail vein injection of intralipid (120 mg/mouse) or recombinant mouse IL-6 (500 $\mathrm{mg} / \mathrm{kg}$ body weight).

Mice were maintained under specific pathogen-free conditions at the Center for Laboratory Animal Science, National Defense Medical College.

Human subjects. Liver tissue samples and serum were collected from 44 NASH patients (age, $46.1 \pm 2.2$ years; 32 men) and 16 NAFL patients (age, $39.2 \pm 1.5$ years; 15 men) when conducting liver biopsies to definitively diagnose NAFLD. Pathological diagnosis of NASH was based on previous reports (40-42). Control liver tissue samples with no steatosis, inflammation, or fibrosis were obtained at hepatectomy from 14 metastatic liver cancer patients (age, $69.1 \pm 2.0$ years; 9 men). Serum samples were collected from 20 healthy control subjects (age, $41.5 \pm 2.4$ years; 17 men).

Human HSC isolation and culture. HSCs were isolated from human liver samples by using the protease/collagenase method as previously described (43). In short, the liver was finely sliced and then stirred for 20 minutes in $100 \mathrm{ml}$ of $0.05 \%$ collagenase/0.5\% protease (Sigma-Aldrich) solution in HBSS (Wako Pure Chemical Industries). After centrifuging the dispersing solution at $400 \mathrm{~g}$ and $4^{\circ} \mathrm{C}$ for 5 minutes, HBSS was added to the precipitate for resuspension. This process was repeated twice, and the precipitate was suspended in $10 \mathrm{ml} \mathrm{HBSS}$. To perform Nycodenz density gradient centrifugation, the cell suspension was layered over 9.0\% Nycodenz solution and centrifuged at 2,800 $\mathrm{g}$ and $4^{\circ} \mathrm{C}$ for 20 minutes. Following centrifugation, the intermediate layer over the $9.0 \%$ Nycodenz solution was collected in DMEM as the HSC fraction and cultured. The present study used only primary cultured HSCs.

Murine HSC isolation and culture. Primary cultured HSCs were isolated from mice and cultured as previously described (15). siRNA was transfected using Lipofectamine RNAiMAX Transfection Reagent (Thermo Fisher Scientific) as previously described (15).

Liver histology and immunohistochemistry. $\mathrm{H} \& \mathrm{E}$ staining and Masson's trichrome (MT) staining were carried out on paraffin-embedded liver sections. For immunohistochemistry, antigens were activated by autoclaving and blocked using Block Ace (Megmilk Snow Brand Co.) followed by primary antibody reaction at room temperature for 4 hours. After washing with PBS, the sections were incubated with Alexa Fluor 488- or Alexa Fluor 647-labeled secondary antibodies (Abcam) at room temperature for 2 hours. Tissue samples were observed under a BX53 microscope (Olympus) and an LSM 710 confocal laser scanning microscope (Carl Zeiss). Double-positive cells were counted in more than 10 high-power fields (magnification, $\times 200$ ). The images were analyzed using ZEN (Carl Zeiss) and ImageJ (NIH).

Preparation and purification of rACLP. Murine rACLP protein was prepared using the Expi293 Expression System (Thermo Fisher Scientific). In short, ExpiFectamine 293 reagent and ACLP-DDK expression vector were used to overexpress ACLP-DDK in Expi293 cells, and the culture medium at 72 hours after transfection was centrifuged at $1,000 \mathrm{~g}$ and $4^{\circ} \mathrm{C}$ for 5 minutes to collect the supernatant, which was concentrated to $100 \mu \mathrm{l}$ using Amicon Ultra-15 Centrifugal Filter Units (100 kDa) (Merck Millipore). The concentrated solution was substituted with TBS (50 mM Tris $\mathrm{HCl}, 150 \mathrm{mM} \mathrm{NaCl}, \mathrm{pH} 7.4$ ), and the volume was adjusted to $10 \mathrm{ml}$. The sample was loaded into a column filled with Anti-FLAG M2 Agarose Affinity Gel (SigmaAldrich). To remove proteins not adsorbed to the gel, the column was washed with $\mathrm{TBS}$, and $0.1 \mathrm{M}$ glycine- $\mathrm{HCl}$ solution ( $\mathrm{pH}$ 3.5) was added to the column to elute the ACLP-DKK protein adsorbed to the gel. The protein concentration of the collected ACLP-DKK protein solution was determined with the BCA method.

The purified ACLP-DKK protein was separated with SDS-PAGE and stained with CBB. Bands around $200 \mathrm{kDa}$ containing ACLP were cut out and digested with trypsin. Using the LTQ Orbitrap XL System (Thermo Fisher Scientific), we performed mano-LC-MS/MS analyses to obtain peptide mass and amino acid sequence. A search with Mascot (Matrix Science) was conducted to identify the protein, which was determined to be ACLP. The purity of the ACLP was confirmed to be $95 \%$ or greater.

Preparation of recombinant mouse FZD8CRD-IgG, mouse FZD8CRD-His, and human LRP6N-IgG proteins. Transfection with mouse FZD8CRD-IgG, mouse FZD8CRD-His, or human LRP6N-IgG expression vector in HEK293 cells was carried out using Lipofectamine LTX (Thermo Fisher Scientific) according to the manufacturer's protocol. IgG Purification Kit-G (Dojindo Molecular Technologies) or Histagged Protein Purification Kit (Medical \& Biological Laboratories) was used to recover the recombinant protein in the culture supernatant at 72 hours after transfection.

$T C F / L E F$ reporter assay. To assess TCF/LEF transcription activity, we used TOPflash and FOPflash luciferase reporter vectors as described (44). Transcriptional induction does not occur in FOP (TOP with a mutation in the TCF/LEF-binding site), even if the WNT pathway is activated. Therefore, if reporter expression that occurs in TOP does not occur in FOP, it indicates that what is occurring is not nonspecific transcription induction, but rather canonical WNT pathway signaling.

HEK293 cells were cultured in a 24-well plate, and Lipofectamine LTX was used to transfect LRP6 expression vector (100 ng/well), FZD8 expression vector (100 ng/well), WNT3A expression vector (200 ng/well), ACLP expression vector (200 ng/well), and either TOPflash or FOPflash luciferase reporter vector. After 36 hours of culturing, intracellular expression of luciferase was quantified using the Dual-Luciferase Reporter Assay System (Promega Corp.). Cells were lysed with a passive lysis buffer included with the kit, Luciferase Assay Reagent II was added to the cell lysate, and chemiluminescence was measured with a TD-20/20 Luminometer (Turner Biosystems).

Western blot analysis. Proteins were extracted from liver tissues or cells using T-PER or M-PER (Thermo Fisher Scientific) including protease inhibitor (Roche), respectively. Nuclear proteins were extracted using NE-PER Nuclear and Cytoplasmic Extraction Kit (Thermo Fisher Scientific). Western blotting was performed as described (15) using Clarity Western ECL Substrate (Bio-Rad) and the ChemiDoc Imaging System (Bio-Rad). Densitometric analysis was performed using ImageQuant TL (GE Healthcare).

Immunoprecipitation. Various combinations of human LRP6N-IgG expression vector (200 ng/well), mouse FZD8CRD-IgG expression vector (200 ng/well), ACLP-DDK expression vector (200 ng/well), and human IgG expression vector were transfected into HEK293 cells cultured in a 6-well plate using Lipofectamine LTX (Thermo Fisher Scientific); cells were recovered 72 hours later. After adding SHE buffer (10 mM HEPES, 210 mM mannitol, 70 mM sucrose, 1 mM EDTA, $1 \mathrm{mM}$ EGTA, $0.15 \mathrm{mM}$ spermine, $0.75 \mathrm{mM}$ spermidine, $\mathrm{pH}$ 7.5) to the cells, they were homogenized using a Potter-Elvehjem Tissue Grinder 
(Wheaton). The homogenate was centrifuged at 3,000 $g$ at $4^{\circ} \mathrm{C}$ for 10 minutes, and the supernatant (cytoplasmic fraction) was recovered. The immunoprecipitation reaction was performed per the manufacturer's protocol; $50 \mu \mathrm{l}$ nProtein A Sepharose 4 Fast Flow (GE Healthcare Life Sciences) was added to $500 \mu \mathrm{l}$ cell extract, followed by inversion mixing at $4^{\circ} \mathrm{C}$ for 2 hours. The liquid mixture was centrifuged at $12,000 \mathrm{~g}$ at $4^{\circ} \mathrm{C}$ for 10 minutes, and the precipitate was suspended in TBS buffer (50 mM Tris, $150 \mathrm{mM} \mathrm{NaCl,} \mathrm{pH} \mathrm{7.5).}$

For analyses in cell-free liquid phase conditions, various combinations of purified mouse ACLP-DDK $(1 \mu \mathrm{g})$, FZD8CRD-IgG $(1 \mu \mathrm{g})$, and human LRP6N-IgG $(1 \mu \mathrm{g})$ were mixed in $300 \mu$ l of binding buffer $(20$ $\mathrm{mM}$ Tris- $\mathrm{HCl}, 100 \mathrm{mM} \mathrm{NaCl}, \mathrm{pH}$ 6.9-7.0, $4^{\circ} \mathrm{C}$ ), followed by inversion mixing at $4^{\circ} \mathrm{C}$ overnight. Immunoprecipitation was carried out with a ProteoTuner Immunoprecipitation Kit (Takara Bio) per the manufacturer's protocol.

Binding assay. Various concentrations of mouse ACLP-DDK or mouse WNT3A, which were biotin-labeled using a Biotin Labeling Kit (Dojindo Molecular Technologies), were inversion-mixed with human FZD8/Fc (final concentration, $5 \mathrm{nM}$ ) in $100 \mu$ l binding buffer (20 mM Tris-HCl, $100 \mathrm{mM} \mathrm{NaCl}, \mathrm{pH} 6.9-7.0,4^{\circ} \mathrm{C}$ ) for 12 hours. The ProteoTuner Immunoprecipitation Kit (Takara Bio) was used per the manufacturer's protocol. Based on biotin measurements with a Sensolyte HABA Biotin Quantification Kit (Anaspec), we determined the amount of ACLP-DDK and WNT3A binding to $1 \mathrm{mg}$ of FZD8/Fc.

Statistics. All values are shown as mean \pm SEM. Statistical analyses were performed using unpaired 2-tailed Student's $t$ test, Mann-Whitney $U$ test, or 1-way ANOVA and Tukey's post hoc test for multiple comparisons. Pearson's correlation was used to compare the relationship between hepatic p-STAT3 and ACLP levels. $P<0.05$ was considered significant.

Study approval. All animal studies were performed with the approval of the National Defense Medical College Animal Use and Care Committee and the Animal Ethics Committee of Keio University. All animals received humane care in compliance with the National
Research Council criteria outlined in the Guide for the Care and Use of Laboratory Animals (National Academies Press, 2011).The present human study was conducted with approval from the institutional review boards of the National Defense Medical College and Keio University School of Medicine. All patients who participated in the present study provided written informed consent prior to the study.

\section{Author contributions}

TT and KT designed the experiments. TT, KT, TS, HF, RI, and MN performed the experiments. TT, KT, HF, RI, JY, TH, SM, TM, YO, $\mathrm{RH}$, and TK were involved in interpretation of data. TT and KT wrote the manuscript.

\section{Acknowledgments}

The authors thank Henry M. Kronenberg (Massachusetts General Hospital and Harvard Medical School) for the generous gift of collagen $1 \alpha 1$ promoter-DKK-1 Tg mice, and Makoto Seo (Saitama Medical University) for the generous gift of an expression vector with a luciferase gene downstream of the human PPAR $\gamma$ promoter. The authors also thank Akira Kikuchi (Osaka University) for helpful discussion and critical comments. This study was supported in part by a Grant-in-Aid for Scientific Research from the Ministry of Education, Culture, Sports, Science, and Technology of Japan (to KT).

Address correspondence to: Kengo Tomita, Division of Gastroenterology and Hepatology, Department of Internal Medicine, National Defense Medical College, 3-2 Namiki, Tokorozawa-shi, Saitama 359-8513, Japan. Phone: 81.4.2995.1211 ext. 2369; Email: kengo@ndmc.ac.jp. Or to: Takanori Kanai, Division of Gastroenterology and Hepatology, Department of Internal Medicine, Keio University School of Medicine, 35 Shinanomachi, Shinjuku-ku, Tokyo 160-8582, Japan. Phone: 81.3.3353.1211 ext. 69344; Email: takagast@z2.keio.jp.
1. Ahmed A, Wong RJ, Harrison SA. Nonalcoholic fatty liver disease review: diagnosis, treatment, and outcomes. Clin Gastroenterol Hepatol. 2015;13(12):2062-2070.

2. Rinella ME. Nonalcoholic fatty liver disease: a systematic review. JAMA. 2015;313(22):2263-2273.

3. Nobili V, et al. Nonalcoholic fatty liver disease: a challenge for pediatricians. JAMA Pediatr. 2015;169(2):170-176.

4. Williams CD, et al. Prevalence of nonalcoholic fatty liver disease and nonalcoholic steatohepatitis among a largely middle-aged population utilizing ultrasound and liver biopsy: a prospective study. Gastroenterology. 2011;140(1):124-131.

5. Day CP, James OF. Steatohepatitis: a tale of two "hits"? Gastroenterology. 1998;114(4):842-845.

6. Tilg H, Moschen AR. Evolution of inflammation in nonalcoholic fatty liver disease: the multiple parallel hits hypothesis. Hepatology. 2010;52(5):1836-1846.

7. Angulo P, et al. Liver fibrosis, but no other histologic features, is associated with long-term outcomes of patients with nonalcoholic fatty liver disease. Gastroenterology. 2015;149(2):389-397.e10.

8. Lee YA, Wallace MC, Friedman SL. Pathobiology of liver fibrosis: a translational success story. Gut.
2015;64(5):830-841.

9. Logan CY, Nusse R. The Wnt signaling pathway in development and disease. Annu Rev Cell Dev Biol. 2004;20:781-810.

10. Prestwich TC, Macdougald OA. Wnt/betacatenin signaling in adipogenesis and metabolism. Curr Opin Cell Biol. 2007;19(6):612-617.

11. Christodoulides C, Lagathu C, Sethi JK, VidalPuig A. Adipogenesis and WNT signalling. Trends Endocrinol Metab. 2009;20(1):16-24.

12. Monga SP. $\beta$-Catenin signaling and roles in liver homeostasis, injury, and tumorigenesis. Gastroenterology. 2015;148(7):1294-1310.

13. Tumelty KE, Layne MD. Adipocyte enhancer binding protein 1 and aortic carboxypeptidaselike protein. In: Rawlings ND, Salvesen G, Woessner JF, eds. Handbook of Proteolytic Enzymes. Cambridge, Massachusetts, USA: Academic Press; 2013:1348-1353.

14. Layne MD, et al. Impaired abdominal wall development and deficient wound healing in mice lacking aortic carboxypeptidase-like protein. $\mathrm{Mol}$ Cell Biol. 2001;21(15):5256-5261.

15. Tomita K, et al. Free cholesterol accumulation in hepatic stellate cells: mechanism of liver fibrosis aggravation in nonalcoholic steatohepatitis in mice. Hepatology. 2014;59(1):154-169.

16. Zhu NL, Wang J, Tsukamoto H. The Necdin-Wnt pathway causes epigenetic peroxisome proliferator-activated receptor gamma repression in hepatic stellate cells. J Biol Chem. 2010;285(40):30463-30471.

17. Yamamoto H, Sakane H, Yamamoto $H$, Michiue T, Kikuchi A. Wnt3a and Dkk1 regulate distinct internalization pathways of LRP6 to tune the activation of beta-catenin signaling. Dev Cell. 2008;15(1):37-48.

18. Sakane H, Yamamoto H, Kikuchi A. LRP6 is internalized by Dkk1 to suppress its phosphorylation in the lipid raft and is recycled for reuse. JCell Sci. 2010;123(Pt 3):360-368.

19. Guo J, et al. Suppression of Wnt signaling by Dkk1 attenuates PTH-mediated stromal cell response and new bone formation. Cell Metab. 2010;11(2):161-171.

20. Lehwald N, Tao GZ, Jang KY, Sorkin M, Knoefel WT, Sylvester KG. Wnt- $\beta$-catenin signaling protects against hepatic ischemia and reperfusion injury in mice. Gastroenterology. 2011;141(2):707-718.e1.

21. Boulter L, et al. Macrophage-derived Wnt opposes Notch signaling to specify hepatic progenitor cell fate in chronic liver disease. Nat Med. 
2012;18(4):572-579.

22. Nam JS, Turcotte TJ, Smith PF, Choi S, Yoon JK. Mouse cristin/R-spondin family proteins are novel ligands for the Frizzled 8 and LRP6 receptors and activate beta-catenin-dependent gene expression. J Biol Chem. 2006;281(19):13247-13257.

23. Wei Q, Yokota C, Semenov MV, Doble B, Woodgett J, He X. R-spondin1 is a high affinity ligand for LRP6 and induces LRP6 phosphorylation and beta-catenin signaling. J Biol Chem . 2007;282(21):15903-15911.

24. Saxena NK, Ikeda K, Rockey DC, Friedman SL, Anania FA. Leptin in hepatic fibrosis: evidence for increased collagen production in stellate cells and lean littermates of ob/ob mice. Hepatology. 2002;35(4):762-771.

25. Meng F, et al. Interleukin-17 signaling in inflammatory, Kupffer cells, and hepatic stellate cells exacerbates liver fibrosis in mice. Gastroenterology. 2012;143(3):765-776.e3.

26. Mashili F, Chibalin AV, Krook A, Zierath JR. Constitutive STAT3 phosphorylation contributes to skeletal muscle insulin resistance in type 2 diabetes. Diabetes. 2013;62(2):457-465.

27. Sato Y, et al. Resolution of liver cirrhosis using vitamin A-coupled liposomes to deliver siRNA against a collagen-specific chaperone. Nat Biotechnol. 2008;26(4):431-442.

28. Kikuchi A, Yamamoto H, Kishida S. Multiplicity of the interactions of Wnt proteins and their receptors. Cell Signal. 2007;19(4):659-671.
29. Clevers H, Nusse R. Wnt/ $\beta$-catenin signaling and disease. Cell. 2012;149(6):1192-1205.

30. Xu Q, et al. Vascular development in the retina and inner ear: control by Norrin and Frizzled-4, a high-affinity ligand-receptor pair. Cell. 2004;116(6):883-895.

31. Naito AT, et al. Complement C1q activates canonical Wnt signaling and promotes aging-related phenotypes. Cell. 2012;149(6):1298-1313.

32. Ross SE, et al. Inhibition of adipogenesis by Wnt signaling. Science. 2000;289(5481):950-953.

33. Yin C, Evason KJ, Asahina K, Stainier DY. Hepatic stellate cells in liver development, regeneration, and cancer. J Clin Invest. 2013;123(5):1902-1910.

34. She H, Xiong S, Hazra S, Tsukamoto H. Adipogenic transcriptional regulation of hepatic stellate cells. J Biol Chem. 2005;280(6):4959-4967.

35. Cohen JC, Horton JD, Hobbs HH. Human fatty liver disease: old questions and new insights. Science. 2011;332(6037):1519-1523.

36. Donnelly KL, Smith CI, Schwarzenberg SJ, Jessurun J, Boldt MD, Parks EJ. Sources of fatty acids stored in liver and secreted via lipoproteins in patients with nonalcoholic fatty liver disease. J Clin Invest. 2005;115(5):1343-1351.

37. Ouchi N, Parker JL, Lugus JJ, Walsh K. Adipokines in inflammation and metabolic disease. Nat Rev Immunol. 2011;11(2):85-97.

38. Schissel SL, Dunsmore SE, Liu X, Shine RW, Perrella MA, Layne MD. Aortic carboxypeptidase-like protein is expressed in fibrotic human lung and its absence protects against bleomycin-induced lung fibrosis. Am J Pathol. 2009;174(3):818-828.

39. Tumelty KE, Smith BD, Nugent MA, Layne MD. Aortic carboxypeptidase-like protein (ACLP) enhances lung myofibroblast differentiation through transforming growth factor $\beta$ receptordependent and -independent pathways. J Biol Chem. 2014;289(5):2526-2536.

40. Younossi ZM, et al. Pathologic criteria for nonalcoholic steatohepatitis: interprotocol agreement and ability to predict liver-related mortality. Hepatology. 2011;53(6):1874-1882.

41. Brunt EM, Kleiner DE, Wilson LA, Belt P, Neuschwander-Tetri BA, NASH Clinical Research Network (CRN). Nonalcoholic fatty liver disease (NAFLD) activity score and the histopathologic diagnosis in NAFLD: distinct clinicopathologic meanings. Hepatology. 2011;53(3):810-820.

42. Matteoni CA, Younossi ZM, Gramlich T, Boparai N, Liu YC, McCullough AJ. Nonalcoholic fatty liver disease: a spectrum of clinical and pathological severity. Gastroenterology. 1999;116(6):1413-1419.

43. Bataller R, et al. Activated human hepatic stellate cells express the renin-angiotensin system and synthesize angiotensin II. Gastroenterology. 2003;125(1):117-125.

44. Wu B, Crampton SP, Hughes CC. Wnt signaling induces matrix metalloproteinase expression and regulates $\mathrm{T}$ cell transmigration. Immunity. 2007;26(2):227-239. 